\title{
Giant vortex and the breakdown of strong pinning in a rotating Bose-Einstein condensate
}

\author{
Amandine Aftalion* $^{*} \quad$ Stan Alama $^{\dagger} \quad$ Lia Bronsard $^{\dagger}$
}

June 22, 2004

\begin{abstract}
We consider a two-dimensional model for a rotating Bose-Einstein condensate (BEC) in an anharmonic trap. The special shape of the trapping potential, negative in a central hole and positive in an annulus, favors an annular shape for the support of the wave function $u$. We study the minimizers of the energy in the Thomas-Fermi limit, where a small parameter $\epsilon$ tends to 0 , for two different regimes of the rotational speed $\Omega$. When $\Omega$ is independent of $\epsilon$, we observe that the energy minimizers acquire vorticity beyond a critical $\Omega$, but the vortices are strongly pinned in the central hole where the potential is negative. In this regime, minimizers exhibit no vortices in the annular bulk of the condensate. There is a critical rotational speed $\Omega=O(|\ln \epsilon|)$ for which this strong pinning effect breaks down and vortices begin to appear in the annular bulk. We derive an asymptotic formula for the critical $\Omega$, and determine precisely the location of nucleation of the vortices at the critical value. These results are related to very recent experimental and numerical observations on BEC.
\end{abstract}

\section{Introduction}

Since the first achievement of BEC in alcali gases in 1995, many properties of these systems are studied experimentally and theoretically, in particular questions concerning vortices. In a BEC, all the atoms are in the same state of lowest energy so that they can be described by the same complex valued wave function, which is at the same time the macroscopic quantum wave function of the condensate and minimizes the so called Gross-Pitaevskii energy. Vortices are thus described by the zeroes of the wave function, around which there is a circulation of phase. A particularity of BEC is that they are trapped systems hence their

*Laboratoire Jacques-Louis Lions \& CNRS, Université Paris-VI, 175 rue du Chevaleret, 75013 Paris, France

${ }^{\dagger}$ Dept. of Mathematics and Statistics, McMaster Univ., Hamilton, Ontario, Canada L8S 4K1. Supported by an NSERC Research Grant. 
geometry depends on the shape of the trapping potential. In most current experiments, this results in a cigar shape condensate.

One way to experimentally obtain vortices is to rotate the trap holding the atoms. If the angular velocity $\Omega$ is large enough, a vortex lattice is observed [ARVK, MCBD, RBD].

We consider here a two-dimensional setting, representing a circular cross-section of a long ellipsoid. We define the energy for the complex-valued wave function $u$ as

$$
\int_{\mathcal{D}}\left\{\frac{1}{2}|\nabla u|^{2}+\frac{1}{4 \epsilon^{2}}\left(|u|^{2}-\bar{a}+V(x)\right)^{2}-\Omega x^{\perp} \cdot(i u, \nabla u)\right\} d x
$$

where $\Omega$ is the angular velocity, $x=\left(x_{1}, x_{2}\right), x^{\perp}=\left(-x_{2}, x_{1}\right), \epsilon>0$ is a small parameter, $V(x)$ is the trapping potential, and the integration takes place over the region $\mathcal{D}=\{x \in$ $\left.\mathbf{R}^{2}: \bar{a}-V(x)>0\right\}$ occupied by the condensate. The constant $\bar{a}$ is chosen such that

$$
\int(\bar{a}-V(x))^{+}=1
$$

We refer to $[\mathrm{ADu}]$ for more details on how this is derived from the physics experiments. In most current experiments, this trapping potential is harmonic, that is $V(x)=x_{1}^{2}+\alpha^{2} x_{2}^{2}$. In this case, the critical angular velocity for nucleation of vortices is of order $|\ln \epsilon|$ (see $[\mathrm{ADu}]$, ) and experiments are limited to angular speeds of the order $\Omega<1 / \epsilon$ when the confinement breaks down. The inhomogeneity of the potential is at the origin of various patterns. For instance, in the 3-D setting it has been observed that vortices bend, and generally do not lie along the axis of rotation (see $[\mathrm{RBD}]$ ). This observation was at the origin of the first mathematical works on BEC, $[\mathrm{AR}, \mathrm{AJ}]$.

In this paper, we focus on very recent experiments in which a laser beam is superimposed upon the magnetic trap holding the atoms. This results in a potential $V(x)$ of a different type [BSSD, SBCD]:

$$
V_{\text {trap }} \simeq(1-b) r^{2}+\frac{k}{4} r^{4}
$$

At a critical value of rotation, the dense vortex lattice gives way to a lattice with hole. Numerical simulations ([KTU] in $2 \mathrm{~d}$ and $[\mathrm{AD}]$ in $3 \mathrm{~d}$ ) illustrate the existence of a multiply quantized "giant vortex."

In the experiments of [BSSD], $b$ is small, so the cross-section is a disc for small values of $\Omega$. Indeed, if $b<1+\left(3 k^{2} / 4\right)^{1 / 3}$, the region determined by $\mathcal{A}=\left\{\bar{a}-V_{\text {trap }}(r)>0\right\}$ is a disc, while if $b>1+\left(3 k^{2} / 4\right)^{1 / 3}$, it is an annulus. When $\Omega$ reaches the order of $1 / \epsilon$ the rotation modifies the potential into $V_{\text {trap }}^{\text {eff }}=V_{\text {trap }}-\epsilon^{2} \Omega^{2} r^{2}$, to effectively increase the value of $b$. The region determined by $\left(\bar{a}-V_{\text {trap }}^{\text {eff }}\right)^{+}$will then become an annulus, creating the "giant vortex". The aim of this paper is to show how the annular topology of the condensate domain affects the 
presence and location of vortices as a function of the angular speed $\Omega$. We concentrate on the effect of the giant vortex on the nucleation of vortices in the condensate domain, rather than on the creation of the hole itself. To do this we choose a trapping potential which ensures the presence of the hole even at low rotational speeds, when individual vortices may be discerned by variational methods. In the sample potential (1.1) this means taking $b>1+\left(3 k^{2} / 4\right)^{1 / 3}$, so then the region $\mathcal{A}=\left\{\bar{a}-V_{\text {trap }}(r)>0\right\}$ will be an annulus when $\Omega \leq O(|\ln \epsilon|)$.

Our results concern both fixed rotation $\Omega$ and rotations which grow with $\epsilon$. When $\Omega$ is fixed, minimizers converge to a non-zero radially equivariant solution away from the hole, which indeed plays the role of a giant vortex, carrying a degree increasing with $\Omega$. We also consider rotations of speed $\Omega=O(|\ln \epsilon|)$ and prove the existence of a critical rotation, above which the strong pinning effect of the giant vortex breaks down. Indeed, above this critical value we observe not only the giant vortex (with vorticity of the order of $|\ln \epsilon|$ ) but also free vortices in the annular bulk. Near the critical $\Omega$ these vortices will concentrate along a precisely defined set $\mathcal{C}$ consisting of finitely many concentric circles in $\mathcal{A}$.

Mathematical Formulation. We model the two-dimensional BEC as a variational problem. We seek minimizers $u=u_{\epsilon} \in H_{0}^{1}(\mathcal{D} ; \mathbf{C})$ of the energy functional

$$
E_{\epsilon}(u)=\int_{\mathcal{D}}\left\{\frac{1}{2}|\nabla u|^{2}-\Omega \mathbf{x}^{\perp} \cdot(i u, \nabla u)+\frac{1}{4 \epsilon^{2}}\left[\left(|u|^{2}-a(x)\right)^{2}-\left(a^{-}(x)\right)^{2}\right]\right\} d x,
$$

where $a(x)=a(r)$ is a smooth radial function in $\mathcal{D}$, a disc of radius $R_{0}$ satisfying:

$(\mathrm{H} 0) a=a(r) \in C^{1}(\mathcal{D})$ is radial, with $a=0$ on $\partial \mathcal{D}$;

(H1) there exists $0<r_{0}<R_{0}$ such that $\mathcal{A}=\left\{x \in \mathbf{R}^{2}: a>0\right\}=B_{R_{0}}(0) \backslash \bar{B}_{r_{0}}(0)$, $T=\{a<0\}=B_{r_{0}}(0) ;$

(H2) There exist constants $\alpha_{0}, \beta_{0}>0$ such that

$$
a(r) \sim \begin{cases}\alpha_{0}\left(r-r_{0}\right), & \text { as } r \sim r_{0} \\ \beta_{0}\left(R_{0}-r\right), & \text { as } r \sim R_{0}\end{cases}
$$

(H3) $\int_{\mathbf{R}^{2}} a^{+}=1$.

We recall that the "anharmonic" potentials given by (1.1) satisfy these hypotheses for $b>$ $1+\left(3 k^{2} / 4\right)^{1 / 3}$. In the limit $\epsilon \rightarrow 0$, energy minimization strongly penalizes the hole $T$ where $a<0$, and we expect the resulting density profile is asymptotically localized in the annular region $\mathcal{A}$.

We now describe our results in greater detail, beginning with rotations $\Omega=O(1)$ : 
The Giant Vortex. The energy minimizers with $\Omega=0$ provide real solutions to the Euler-Lagrange equations. When $\Omega=0, E_{\epsilon}(\eta)=J_{\epsilon}(\eta)$, where

$$
J_{\epsilon}(\eta)=\int_{\mathcal{D}}\left\{\frac{1}{2}|\nabla \eta|^{2}+\frac{1}{4 \epsilon^{2}}\left[\left(|\eta|^{2}-a(x)\right)^{2}-\left(a^{-}(x)\right)^{2}\right]\right\} d x .
$$

The minimizer $\eta_{\epsilon}$ of $J_{\epsilon}$ is (up to a complex multiplier of modulus one) the unique positive solution of

$$
\Delta \eta_{\epsilon}+\frac{1}{\epsilon^{2}} \eta_{\epsilon}\left(a(x)-\eta_{\epsilon}^{2}\right)=0 \quad \text { in } \mathcal{D}, \quad \eta_{\epsilon}=0 \quad \text { on } \partial \mathcal{D} .
$$

Moreover $\eta_{\epsilon}^{2}$ converges to $a^{+}$in $L^{2}(\mathcal{D})$ and uniformly on any compact set of $\mathcal{A}$ or $T$. (See Proposition 2.1).

By a remarkable identity (see Lassoued \& Mironescu [LM]), the energy $E_{\epsilon}$ splits into two parts, the energy $J_{\epsilon}\left(\eta_{\epsilon}\right)$ of the density profile and a reduced energy of the complex phase $v=u / \eta_{\epsilon}$, which allows us to compute the limiting energy and identify the size of the giant vortex in $T$ :

Theorem 1.1 Let $\Omega$ be fixed, and let $D_{0} \in \mathbf{Z}$ be the minimizer of

$$
g_{0}(d)=\frac{1}{2} \Lambda_{1} d^{2}-\Omega d, d \in \mathbf{Z}, \quad \text { where } \Lambda_{1}=\int_{\mathcal{A}} \frac{a}{|x|^{2}} .
$$

Let $u_{\epsilon}$ be a sequence of minimizers of $E_{\epsilon}$, then

(i) $E_{\epsilon}\left(u_{\epsilon}\right)-E_{\epsilon}\left(\eta_{\epsilon}\right) \rightarrow g_{0}\left(D_{0}\right)$, as $\epsilon$ tends to 0 ,

(ii) There exists a subsequence $\epsilon \rightarrow 0$ and $\alpha \in \mathbf{C}$ with $|\alpha|=1$ so that

$$
\frac{u_{\epsilon}}{\eta_{\epsilon}} \rightarrow \alpha e^{i D_{0} \theta} \quad \text { in } H_{l o c}^{1}(\mathcal{A}) \text {, and } \quad\left|\frac{u_{\epsilon}}{\eta_{\epsilon}}\right| \rightarrow 1, \text { locally uniformly in } \mathcal{A} .
$$

(iii) for every fixed $r$ such that $\partial B_{r}(0) \subset \mathcal{A}$, deg $\left(\frac{u_{\epsilon}}{\eta_{\epsilon}}, \partial B_{r}\right)=D_{0}$ for $\epsilon$ sufficiently small.

(iv) $|u(x)| \rightarrow 0$ locally uniformly in $T$ and

$$
|u(x)| \leq C \epsilon^{1 / 6} \exp \left[-\operatorname{dist}(x, \partial T) / \epsilon^{2 / 3}\right]
$$

for all $x \in T$ with $\operatorname{dist}(x, \partial T) \geq \epsilon^{1 / 3}$.

Thus, we observe a sequence of phase transitions as $\Omega$ increases, at which the minimizer of $g_{0}(d)$ jumps from one degree to another, and the giant vortex takes on larger and larger vorticity, while there are no vortices in the annulus.

We also consider another way to model the presence of the hole. In previous works on BEC (see $[\mathrm{AJ}, \mathrm{ADu}, \mathrm{AR}]$ for example) the assumption has been made that the potential 
well completely determines the shape of the condensate. The corresponding analysis leads here to a zero Dirichlet boundary condition on $\partial \mathcal{A}$, and the energy

$$
\hat{E}_{\epsilon}(u):=\int_{\mathcal{A}}\left\{\frac{1}{2}|\nabla u|^{2}-\Omega \mathbf{x}^{\perp} \cdot(i u, \nabla u)+\frac{1}{4 \epsilon^{2}}\left(|u|^{2}-a(x)\right)^{2}\right\} d x, \quad u \in H_{0}^{1}(\mathcal{A} ; \mathbf{C}) .
$$

We will see that this model and (1.2) give very similar results, which is to be expected as minimizers of (1.2) vanish exponentially to zero in the hole $T$ (see Lemma 2.5).

Vortices in the annulus. In the second part of the paper, we consider the case when $\Omega=O(|\ln \epsilon|)$, and thus vortices are nucleated in the bulk $\mathcal{A}$ of the condensate. For simplicity, we restrict to the model in the annulus $\mathcal{A}$, that is $(1.7)$ with $u \in H_{0}^{1}(\mathcal{A} ; \mathbf{C})$ having a zero Dirichlet condition on $\partial A$. As in the previous part, we decouple the modulus and phase of $u_{\epsilon}=\hat{\eta}_{\epsilon} v_{\epsilon}$, where now $\hat{\eta}_{\epsilon} \in H_{0}^{1}(\mathcal{A}, \mathbf{R})$ minimizes the energy $\hat{J}_{\epsilon}$ retricted to real-valued functions with Dirichlet condition on $\partial \mathcal{A}$. We assume a specific asymptotic form for the rotation $\Omega$,

$$
\Omega=\omega_{0}|\ln \epsilon|+\omega_{1} \ln |\ln \epsilon| .
$$

It is well-known in the Ginzburg-Landau model that vortices become energetically favorable at a critical value of the rotation $\Omega^{*}=O(|\ln \epsilon|)$ (see [Se2], for example). We show that the same general principle holds in the annular case, but with an interesting difference. The hole acts genuinely as a giant vortex, and exerts a repulsive force on "free" vortices in the interior of $\mathcal{A}$ which effectively balances the force of the rotation. Hence the vortices are going to lie on a specific circle.

We identify vortices using energy concentration, via the construction of "vortex balls" and the consequent lower bounds derived by Sandier \& Serfaty [SSe3]. For technical reasons we must excise a thin neighborhood of a certain width $\rho=\rho_{\epsilon}$ with

$$
(\ln |\ln \epsilon|)^{-1 / 2}<<\rho<<1
$$

from the two edges of the annulus and restrict our analysis to vortices lying in the interior region

$$
\mathcal{A}_{\rho}=\left\{x \in \mathcal{A}, \operatorname{dist}(x, \partial \mathcal{A})>\rho_{\epsilon}\right\} .
$$

There may well exist vortices near the edges of $\mathcal{A}$, but the value of $a$ being very small near $\partial \mathcal{A}$ we have no way of controlling these outlying vortices. We find that there exists a finite number of balls $B_{i} \subset \mathcal{A}_{\rho}$ with vanishingly small total radii so that $\left|v_{\epsilon}\right|$ is very close to 1 in $\mathcal{A}_{\rho} \backslash \cup B_{i}$. Each vortex ball carries an associated integer winding number $d_{i}=\operatorname{deg}\left(\frac{v}{|v|}, \partial B_{i}\right)$. We say that $u_{\epsilon}$ has an essential vortex at $p_{i} \in \mathcal{A}_{\rho}$ if there is a vortex ball $B_{i}$ centered at $p_{i}$ with degree $d_{i} \neq 0$.

We prove the following result: 
Theorem 1.2 Assume a(x) satisfies hypotheses (H0)-(H3) in $\mathcal{A}$, and

(H4) $\quad a(x)$ is a real-analytic function of $r=|x|$.

Let $\rho=\rho_{\epsilon}, \mathcal{A}_{\rho}$ satisfy (1.9)-(1.10). Then, there exist constants $\omega_{0}^{*}, \omega_{1}^{*}$ such that if $u_{\epsilon}$ is a sequence of minimizers of $\hat{E}_{\epsilon}$ in $H_{0}^{1}(\mathcal{A})$, there exists a finite collection of disjoint balls $B_{i}$ of small radius such that $\operatorname{deg}\left(u_{\epsilon} /\left|u_{\epsilon}\right|\right)=d_{i}$ and the following holds:

(i) if either $\omega_{0}<\omega_{0}^{*}$ or $\omega_{0}=\omega_{0}^{*}$ and $\omega_{1}<-\omega_{1}^{*}$, then for all $\epsilon$ sufficiently small $u_{\epsilon}$ has no essential vortices in $\mathcal{A}_{\rho}: \sum_{\mathcal{A}_{\rho}}\left|d_{i}\right|=0$.

(ii) if $\omega_{0}=\omega_{0}^{*}$ and $\omega_{1}>-\omega_{1}^{*}$, then for all $\epsilon$ sufficiently small, any essential vortex in $\mathcal{A}_{\rho}$ has positive degree and is localized on a set $\mathcal{C}$ of concentric circles in the following sense: there exists an $M \in \mathbf{N}$ such that if $p_{i}$ are the centers of $B_{i}$,

$$
\sum_{\substack{p_{i} \in \mathcal{A}_{\rho} \\ d_{i}<0}}\left|d_{i}\right|+\sum_{\substack{p_{i} \in \mathcal{A}_{\rho} \\ \operatorname{dist}\left(p_{i}, \mathcal{C}\right)>\\[\ln \epsilon]^{-1 / 2 M}}}\left|d_{i}\right|=0
$$

for all $\epsilon$ sufficiently small. Moreover, the total degree in $\mathcal{A}_{\rho}$,

$$
\mathbf{D}_{\epsilon}:=\sum_{p_{i} \in \mathcal{A}_{\rho}}\left|d_{i}\right|=\sum_{\substack{\mathrm{dist}\left(p_{i}, \mathcal{C}\right)<\\[\ln \epsilon]^{-1 / 2 M}}} d_{i} \leq C \ln |\ln \epsilon|
$$

for $C$ independent of $\epsilon$. If $\omega_{1}>0$, then $\mathbf{D}_{\epsilon} \geq 1$.

(iii) there exists $\omega_{1}^{\#}>0$ so that whenever $\omega_{1} \geq \omega_{1}^{\#}$ and $\omega_{0}=\omega_{0}^{*}$, then $\mathbf{D}_{\epsilon} \geq c \ln |\ln \epsilon|$ for $c$ independent of $\epsilon$.

We emphasize that the sum of degrees is taken over the vortex balls in $\mathcal{A}_{\rho}$.

This Theorem relies on an asymptotic development of the energy. We use the resulting "vortexless" configuration $\eta_{\epsilon} e^{i D_{\epsilon} \theta}$ as a background configuration in much the same way as the Meissner solution is used to study the critical fields of a superconductor in [Se2, SSe1, SSe4]. (A related problem of vortices in rotating superfluid is solved in [Se2], where the parallel between rotations and magnetic fields also appears.) Indeed, our asymptotic expansion of the energy leads to the appearance of a new potential function,

$$
F(r):=\int_{r}^{R_{0}} a(s)\left(s-\frac{1}{\Lambda_{1} s}\right) d s, \quad r \in\left[r_{0}, R_{0}\right] .
$$

We have $F(r) \geq 0, F\left(r_{0}\right)=0=F\left(R_{0}\right)$, and

$$
\max _{r \in\left[r_{0}, R_{0}\right]} \frac{F(r)}{a(r)}=: K_{0}>0,
$$


is attained on a finite set of radii $\Lambda \subset\left(r_{0}, R_{0}\right)$. (See Lemma 4.2). We show that $\omega_{0}^{*}=\frac{1}{2 K_{0}}$ gives the desired critical value of rotation, in the sense that when $\omega_{0}<\omega_{0}^{*}$ minimizers will have no essential vortices inside $\mathcal{A}$. On the other hand, if $\omega_{0}=\omega_{0}^{*}$ and $\omega_{1}>0$, vortices converge to the set $\mathcal{C}$ of concentric circles of radii $r^{*} \in \Gamma$ as $\epsilon \rightarrow 0$.

The lower bound on the energy leads to the upper bound on the number of essential vortices, the positivity of the degrees, and their location in $\mathcal{A}_{\rho}$. The lower bound on the number of vortices comes from a better upper bound construction with vortices distributed along the optimal set $\mathcal{C}$ when $\omega_{1}$ is large.

We conjecture that the vortices are evenly distributed on the circles $\mathcal{C}$, and as $\epsilon \rightarrow 0$ their normalized density measure converges to a constant times the arclength measure on $\mathcal{C}$. (See for instance Sandier \& Soret [SSo] for a related result.) We are not able to show this, as it requires sharper information on the coefficients of $\ln |\ln \epsilon|$ in the lower bound expansion.

Mathematical perspective. Variational problems of a similar type to (1.7) with spatially varying coefficients have also been introduced to model vortex pinning due to material inhomogeneities in a superconductor. Most analytical results have concentrated on the case where $a(x)>0$ in $\Omega$ (see [AnSh1],[L].) These authors look at the Dirichlet problem, where a topologically nontrivial boundary function induces vortices, rather than the rotation $\Omega$ as in our $\mathcal{E}_{\epsilon}$. They show that vortices are attracted to the minima of $a(x)$, the "pinning sites". In the case of superconductors, the same pinning effect of the minima of $a$ was noted by Aftalion, Sandier, \& Serfaty [ASSe]. There, the external magnetic field plays an analogous role to $\Omega$ in generating vortices, and they observe that the effect of such inhomogeneities is to vary the critical field (at which vortices first appear) at order $|\ln \epsilon|$. A recent paper of André, Bauman, \& Phillips [AnBaPh] treats the case where $a(x)$ is allowed to vanish. In contrast to our setting, in $[\mathrm{AnBaPh}]$, the pinning potential $a(x)$ is nonnegative and vanishes at a finite number of points. They show that when the applied field (which plays the role of our angular speed $\Omega$ ) is large but fixed (independent of $\epsilon$ ), minimizers may have non-zero degree yet do not experience loss of superconductivity in the interior of the region where $a>0$ : in the limit $\epsilon \rightarrow 0$ the superconducting density $|u|^{2} \rightarrow a(x)$ locally in $H^{1}$. The vortices are pinned to the zeros of $a$, and none appear in the region where $a>0$. In their result, it is important that $\sqrt{a}$ is in $H^{1}$, which is not the case here. Note that our hypothesis on the potential imply that $a(r)$ vanishes linearly near $\partial \mathcal{A}$, and hence (unlike $[\mathrm{AnBaPh}]$ ) $\sqrt{a^{+}}$is not in $H_{0}^{1}(\mathcal{A})$. In particular, the profile of the condensate is singular near the boundary, and contributes to a divergent term in the expansion of energy. We overcome this difficulty by a splitting of the energy, inspired by [LM], to separate the contribution of the vortices from that of the singular boundary layer. 
Ginzburg-Landau models in domains with holes have also been studied by other authors ([RuSt], [JiMo]) but in the context of "permanent currents", locally minimizing solutions with zero applied field (rotation). Like our giant vortex states, these represent a particular homotopy class of the bulk domain, and introduce vorticity without vortices. An important difference though is that their solutions are only locally minimizing, while our solutions are global minimizers, with vorticity selected by the external rotation $\Omega$.

The paper is organized as follows: in section 2, we prove general results about the minimizers of $J_{\epsilon}$ and behaviour of $u_{\epsilon}$ in $T$. Then section 3 is devoted to the proof of Theorem 1.1 and section 4 to theorem 1.2. Finally, at the end of section 4 , we explain the case where $a=1$.

\section{Preliminaries}

In this section, we present some preliminary results which we will require throughout the paper. First, we study the real solutions $\eta_{\epsilon}$, since they give, in some sense, the density profile of vortexless configurations and define the underlying shape of the condensate. Their energy diverges with $\epsilon$, and we decompose the energy in order to decouple the profile from the contribution of vortices. Finally, we compare two different models for the hole (or Giant Vortex).

\subsection{Determining the density profile}

Firstly, we want to determine the profile of the solution.

Proposition 2.1 Problem (1.4) admits a unique positive solution $\eta_{\epsilon}$, which is the unique minimizer of $J_{\epsilon}$ in $H_{0}^{1}(\mathcal{D})$ up to a complex multiplier of modulus one. In addition,

(i) $\eta_{\epsilon} \in C^{\infty}(\mathcal{D})$ is radial;

(ii) $0<\eta_{\epsilon}(x) \leq \max _{\mathcal{D}} a$, and $\left|\nabla \eta_{\epsilon}\right| \leq C / \epsilon$;

(iii) $J_{\epsilon}\left(\eta_{\epsilon}\right) \leq C|\ln \epsilon|$ and $J_{\epsilon}\left(\eta_{\epsilon}\right)$ is bounded in $L_{l o c}^{\infty}(\mathcal{A})$ and $L_{l o c}^{\infty}(T)$.

(iv) There exists a constant $C$ independent of $\epsilon$ so that

$$
\left|\eta_{\epsilon}(x)-\sqrt{a^{+}(x)}\right| \leq C \epsilon^{1 / 3} \sqrt{a^{+}(x)} \text { for every } x \in \mathcal{A} \text { with } \operatorname{dist}(x, \partial \mathcal{A}) \geq \epsilon^{1 / 3}
$$

(v) $0<\eta_{\epsilon}(x) \leq C \epsilon^{1 / 6} \exp \left[-\operatorname{dist}(x, \partial T) / \epsilon^{2 / 3}\right]$ for every $x \in T$ with $\operatorname{dist}(x, \partial T) \geq \epsilon^{1 / 3}$; where $C>0$ is a constant independent of $\epsilon$. 
In particular, $(v)$ implies that $\eta_{\epsilon} \rightarrow 0$ locally uniformly in the hole $T$. The assertion $(i v)$ implies that $\left|\eta_{\epsilon}^{2}(x)-a^{+}(x)\right|$ is small with respect to $a^{+}(x)$ itself provided we remain at a small distance $\left(\epsilon^{1 / 3}\right)$ from the boundary of $\mathcal{A}$; this will be essential in our analysis of the nucleation of vortices into the annulus $\mathcal{A}$ with increasing rotation.

Remark 2.2 We note that the problem in the annulus $\mathcal{A}$ has its own real minimizer, $\hat{\eta}_{\epsilon} \in$ $H_{0}^{1}(\mathcal{A} ; \mathbf{R})$, which satisfies conclusions (i)-(iv) of Proposition 2.1.

Remark 2.3 We also have $\eta_{\epsilon}^{2} \rightarrow a^{+}$in $C_{\text {loc }}^{1, \alpha}(T)$ and $C_{\text {loc }}^{1, \alpha}(\mathcal{A}),\left\|\eta_{\epsilon}-\sqrt{a}\right\|_{C^{1}(K)} \leq C_{K} \epsilon^{2}$, for any compact subset $K$ of $T$ or $\mathcal{A}$.

Proof of Proposition 2.1: The existence of a positive minimizer of $J_{\epsilon}$ in $H_{0}^{1}(\mathcal{D})$ is standard. Since $J_{\epsilon}(|\eta|) \leq J_{\epsilon}(\eta)$, with equality if and only if $\eta=|\eta| e^{i \alpha}$, it implies that the minimizer is a real positive function, which satisfies the Euler Lagrange equation, up to multiplication by a number of modulus 1 . The uniqueness comes from $[\mathrm{BrO}]$. Let us recall the proof briefly. If $\xi$ and $\eta$ are two solutions, then $w=\xi / \eta$ satisfies an equation, that we multiply by $w-1$ and integrate in $\mathcal{D}$ to obtain that $w \equiv 1$.

$(i)$ : by the uniqueness, $\eta$ must be radial.

(ii): The Maximum Principle yields that $\eta>0$ in $\mathcal{D}$ and $\eta<\max _{A} a$. The estimate on the gradient follows from the equation and Gagliardo-Nirenberg inequality as in $[\mathrm{BBH}]$.

(iii): since $\eta_{\epsilon}$ is the minimizer of $J_{\epsilon}$, we just need to construct a test function for which we have a bound on the energy. We define $\xi(r)=\gamma\left(a^{+}(r)\right)$, where

$$
\gamma(s)= \begin{cases}\sqrt{s}, & \text { if } s>\epsilon^{2 / 3} \\ \frac{s}{\epsilon^{1 / 3}}, & \text { if } s<\epsilon^{2 / 3}\end{cases}
$$

Using the coarea formula, we find

$$
\int_{\mathcal{D}}|\nabla \xi|^{2}=\int_{r_{0}}^{R_{0}} \gamma^{\prime}\left(a^{+}(r)\right)^{2}\left|\nabla a^{+}\right|^{2} d r \leq C \int_{0}^{\bar{a}} \gamma^{\prime}(s)^{2} d s \leq C|\ln \epsilon| .
$$

For the other term,

$$
\int_{\mathcal{D}}\left(a^{+}-\gamma\left(a^{+}\right)^{2}\right)^{2} d r \leq \int_{0}^{\epsilon^{2 / 3}}\left(s-\gamma(s)^{2}\right)^{2} d s \leq C \epsilon^{2} .
$$

Hence, the energy of this test function is bounded by $|\ln \epsilon|$.

In order to get the energy bound on compact sets, we follow [LM] and fix $\delta$ such that $K_{\delta}=\{x \in \mathcal{A}$, dist $(x, \partial K)<\delta\}$ is included in $\mathcal{A}$. We have in particular $J_{\epsilon}\left(\eta_{\epsilon}, K_{\delta} \backslash K\right) \leq$ $C|\ln \epsilon|$. Hence there exists a compact $K^{\prime}$ containing $K$ such that $J_{\epsilon}\left(\eta_{\epsilon}, \partial K^{\prime}\right) \leq C^{\prime}|\ln \epsilon|$. We 
can assume that $K^{\prime}=\left(r_{0}, r_{1}\right)$ and let $K_{\epsilon}^{\prime}=\left(r_{0}+\epsilon, r_{1}-\epsilon\right)$. We consider the following test function

$$
v_{\epsilon}= \begin{cases}\eta_{\epsilon}, & \text { in } \mathcal{D} \backslash \bar{K}^{\prime}, \\ \sqrt{a}, & \text { in } K_{\epsilon}^{\prime}, \\ \sqrt{t \eta_{\epsilon}^{2}\left(r_{0}\right)+(1-t) a\left(r_{0}+\epsilon\right)}, & \text { if } r \in\left(r_{0}, r_{0}+\epsilon\right) \text { and } a(r)=(1-t) a\left(r_{0}+\epsilon\right)+t a\left(r_{0}\right), \\ \sqrt{t \eta_{\epsilon}^{2}\left(r_{1}\right)+(1-t) a\left(r_{1}-\epsilon\right)}, & \text { if } r \in\left(r_{1}-\epsilon, r_{1}\right) \text { and } a(r)=(1-t) a\left(r_{1}-\epsilon\right)+t a\left(r_{1}\right),\end{cases}
$$

Using that $J_{\epsilon}\left(\eta_{\epsilon}\right) \leq J_{\epsilon}\left(v_{\epsilon}\right)$ and that $v_{\epsilon}$ and $\eta_{\epsilon}$ are equal in $\mathcal{D} \backslash \bar{K}^{\prime}$, we obtain $J_{\epsilon}\left(\eta_{\epsilon}, K^{\prime}\right) \leq$ $J_{\epsilon}\left(v_{\epsilon}, K^{\prime}\right)$. A computation of $J_{\epsilon}\left(v_{\epsilon}, K^{\prime}\right)$ together with the hypothesis $J_{\epsilon}\left(\eta_{\epsilon}, \partial K^{\prime}\right) \leq C^{\prime}|\ln \epsilon|$ gives the result.

A similar proof holds in $T$ and yields in fact a bound in $\epsilon|\ln \epsilon|$ for the energy in every compact subset of $T$.

(iv): Let $x_{0} \in \mathcal{A}$ with $\operatorname{dist}\left(x_{0}, \mathcal{A}\right)>\rho$, and $\delta<\rho$. We will construct a subsolution $w$ and a supersolution $W$ to (1.4) in $B_{\delta}\left(x_{0}\right)$ with $\left.w\right|_{\partial B_{\delta}\left(x_{0}\right)} \leq\left.\eta_{\epsilon}\right|_{\partial B_{\delta}\left(x_{0}\right)} \leq\left. W\right|_{\partial B_{\delta}\left(x_{0}\right)}$. By uniqueness of solutions of (1.4), we conclude that $w \leq \eta_{\epsilon} \leq W$ in $B_{\delta}\left(x_{0}\right)$.

Let

$$
A=\max _{B_{\delta}\left(x_{0}\right)} a, \quad \alpha=\min _{B_{\delta}\left(x_{0}\right)} a, \quad M=\max _{\mathcal{D}} a .
$$

By hypothesis (H2) on $a$, we have $\alpha \geq c \rho$ for $\rho$ sufficiently small. For our supersolution, we take:

$$
W(x):=\sqrt{A} \operatorname{coth}\left(\operatorname{coth}^{-1}\left(\sqrt{\frac{M}{A}}\right)+\frac{\delta^{2}-\left|x-x_{0}\right|^{2}}{3 \delta \epsilon} \sqrt{A}\right) .
$$

As in [AnSh], $W$ is such that $\Delta W+\frac{1}{\epsilon^{2}}\left(A-W^{2}\right) W \leq 0$. Since $W=\sqrt{M} \geq \eta_{\epsilon}$ on $\partial B_{\delta}\left(x_{0}\right)$, we have $\eta_{\epsilon}(x) \leq W(x)$ in $B_{\delta}\left(x_{0}\right)$. In particular,

$$
0 \leq W\left(x_{0}\right)-\sqrt{A} \leq \sqrt{A}\left[\operatorname{coth}\left(\frac{\delta \sqrt{A}}{3 \epsilon}\right)-1\right] \leq \sqrt{A} \exp \left(-\frac{2 \delta \sqrt{A}}{3 \epsilon}\right) .
$$

Since $a$ is smooth in $\mathcal{A}$, we may approximate $a\left(x_{0}\right)$ by $A$ making a small error: $\mid \sqrt{a\left(x_{0}\right)}-$ $\sqrt{A} \mid \leq C \delta / \sqrt{A} \leq C \delta / \rho^{1 / 2}$ with constant $C$ depending only on the $C^{1}$ norm of $a$ in $\mathcal{A}$. Hence,

$$
\frac{W\left(x_{0}\right)-\sqrt{a\left(x_{0}\right)}}{\sqrt{a\left(x_{0}\right)}} \leq \frac{\sqrt{A}-\sqrt{a\left(x_{0}\right)}}{\sqrt{a\left(x_{0}\right)}}+\frac{\sqrt{A}}{\sqrt{a\left(x_{0}\right)}} e^{\left(-\frac{\delta \sqrt{A}}{\epsilon}\right)} \leq C\left(\frac{\delta}{\rho^{1 / 2}}+e^{\left(-\frac{2 \delta \sqrt{A}}{3 \epsilon}\right)}\right) .
$$

We now choose $\delta=\epsilon^{2 / 3}$ and $\rho=\epsilon^{1 / 3}$. Then the second term of (2.2) is exponentially small compared to the first, and we obtain

$$
\frac{\eta_{\epsilon}\left(x_{0}\right)-\sqrt{a\left(x_{0}\right)}}{\sqrt{a\left(x_{0}\right)}} \leq \frac{W\left(x_{0}\right)-\sqrt{a\left(x_{0}\right)}}{\sqrt{a\left(x_{0}\right)}} \leq C \epsilon^{1 / 3}
$$


for all $x_{0} \in \mathcal{A}$ with $\operatorname{dist}\left(x_{0}, \partial \mathcal{A}\right) \geq \epsilon^{2 / 3}$.

To construct a subsolution in $B_{\delta}\left(x_{0}\right)$, we first let $\tilde{w}(x)$ be the solution of

$$
-\Delta \tilde{w}+\frac{1}{\tilde{\epsilon}^{2}} \tilde{w}\left(\tilde{w}^{2}-1\right)=0, \quad \text { in } B_{1}(0), \quad \tilde{w}=0, \quad \text { on } \partial B_{1}(0),
$$

and $\tilde{\epsilon}=\epsilon / \delta \sqrt{\alpha}$. This problem has been studied in [Se2], where the exponential decay estimate is derived: $0 \leq 1-\tilde{w}(\tilde{x}) \leq C \exp \left(-\frac{1-|x|^{2}}{2 \tilde{\epsilon}}\right)$. Then we map this function to $B_{\delta}\left(x_{0}\right)$ via $\tilde{x}=\frac{x-x_{0}}{\delta}$,

$$
w(x)=\sqrt{\alpha} \tilde{w}\left(\frac{x-x_{0}}{\delta}\right) .
$$

We have

$$
\left\{\begin{array}{c}
-\Delta w+\frac{1}{\epsilon^{2}}\left(w^{2}-a(x)\right) w \leq-\Delta w+\frac{1}{\epsilon^{2}}\left(w^{2}-\alpha\right) w=0, \text { in } B_{\delta}\left(x_{0}\right), \\
w=0<\eta_{\epsilon} \text { on } \partial B_{\delta}\left(x_{0}\right),
\end{array}\right.
$$

By the decay estimate (2.3) we obtain $0 \leq \sqrt{\alpha}-w\left(x_{0}\right) \leq \sqrt{\alpha} \exp \left(-\frac{\delta \sqrt{\alpha}}{2 \epsilon}\right)$. Arguing as in (2.2), we approximate $\sqrt{\alpha}$ by $\sqrt{a\left(x_{0}\right)}$ in the estimate, and choose $\delta=\epsilon^{2 / 3}, \rho=\epsilon^{1 / 3}$. We obtain:

$$
\frac{\sqrt{a\left(x_{0}\right)}-\eta_{\epsilon}\left(x_{0}\right)}{\sqrt{a\left(x_{0}\right)}} \leq \frac{\sqrt{a\left(x_{0}\right)}-w\left(x_{0}\right)}{\sqrt{a\left(x_{0}\right)}} \leq C \frac{\delta}{\rho^{1 / 2}}=C \epsilon^{1 / 3}
$$

for all $x_{0} \in \mathcal{A}$ with dist $\left(x_{0}, \partial \mathcal{A}\right) \geq \epsilon^{2 / 3}$. This proves the desired estimate for $\eta_{\epsilon}$.

$(v)$ : this is a special case of Proposition 2.5 below. $\diamond$

Remark 2.4 Except for (i), Proposition 2.1 holds even if a is not radial.

\subsection{Comparing two models for the Giant Vortex}

In the hole $T=B_{r_{0}}(0), a<0$ and the potential energy of $u$ is coercive, so we expect that, in the singular limit $\epsilon \rightarrow 0, u \rightarrow 0$ in $T$. Therefore it is natural to try to model the apparent hole by a domain wall on $\partial T$, and impose a zero Dirichlet condition on $u$ : let

$$
\hat{E}_{\epsilon}(u):=E_{\epsilon}(u ; A)=\int_{\mathcal{A}}\left\{\frac{1}{2}|\nabla u|^{2}-\Omega x^{\perp} \cdot(i u, \nabla u)+\frac{1}{4 \epsilon^{2}}\left(|u|^{2}-a\right)^{2}\right\},
$$

defined for $u \in H_{0}^{1}(\mathcal{A})$. The following Proposition demonstrates that minimizers for these two energies should be qualitatively similar; in fact, all of the results proven in the remainder of the paper for $E_{\epsilon}$ may be modified in a transparent way to hold in the same form for $\hat{E}_{\epsilon}$, and hence the two minimization problems describe essentially the same phenomena. 
Proposition 2.5 Assume $\Omega \leq C_{0}|\ln \epsilon|$ for some constant $C_{0}>0$. Then, for any minimizer $u$ of $E_{\epsilon}$ in $H_{0}^{1}(\mathcal{D})$,

$$
\int_{\mathcal{A}}\left(|u|^{2}-a\right)^{2}+\int_{T}|u|^{4} \leq C_{1} \epsilon^{2}|\ln \epsilon|^{2},
$$

with constant $C_{1}$ depending on $C_{0}$. Moreover, $|u(x)| \rightarrow 0$ locally uniformly in $T$ and

$$
|u(x)| \leq C \epsilon^{1 / 6} \exp \left[-\operatorname{dist}(x, \partial T) / \epsilon^{2 / 3}\right]
$$

for all $x \in T$ with $\operatorname{dist}(x, \partial T) \geq \epsilon^{1 / 3}$.

Proof: We obtain a simple upper bound on the energy $E_{\epsilon}$ by substituting the test function $\eta_{\epsilon}, \min _{H_{0}^{1}(\mathcal{D})} E_{\epsilon} \leq C|\ln \epsilon|$. Note that in $T$,

$$
\left(|u|^{2}-a\right)^{2}-\left(a^{-}\right)^{2}=|u|^{4}+2 a^{-}|u|^{2} \geq|u|^{4} .
$$

We estimate the rotation term by

$$
\begin{aligned}
\left|\int_{\mathcal{D}} \Omega x^{\perp} \cdot(i u, \nabla u)\right| & \leq \frac{1}{2} \int_{\mathcal{D}}|\nabla u|^{2}+\frac{\Omega^{2}}{2} \int_{T}|x|^{2}|u|^{2}+\frac{\Omega^{2}}{2} \int_{\mathcal{A}}|x|^{2}\left[\left(|u|^{2}-a\right)+a\right] \\
& \leq \frac{1}{2} \int_{\mathcal{D}}|\nabla u|^{2}+\frac{\Omega^{2}}{4} \int_{T}|u|^{4}+\frac{\Omega^{2}}{4} \int_{\mathcal{A}}\left(|u|^{2}-a\right)^{2}+C \Omega^{2} .
\end{aligned}
$$

For $\epsilon$ sufficiently small, $\Omega^{2}<<\frac{1}{\epsilon^{2}}$, and hence these terms may be absorbed into the coercive terms in the energy to obtain

$$
C|\ln \epsilon| \geq E_{\epsilon}(u) \geq \frac{1}{4}\left(\frac{1}{\epsilon^{2}}-\Omega^{2}\right)\left(\int_{T}|u|^{4}+\int_{\mathcal{A}}\left(|u|^{2}-a\right)^{2}\right)-C \Omega^{2} .
$$

The estimate (2.4) then follows from the hypothesis $\Omega \leq C|\ln \epsilon|$.

We now turn to the proof of the pointwise convergence in the hole $T$. Any minimizer solves the equation

$$
-\Delta u-2 i \Omega x^{\perp} \cdot \nabla u=\frac{1}{\epsilon^{2}}\left(a(x)-|u|^{2}\right) u
$$

pointwise in $\mathcal{D}$. Let $T_{\epsilon}^{\prime}:=\left\{x \in T: a(x) \leq-\frac{1}{2} \epsilon^{1 / 3}<0\right\}$, so in $T_{\epsilon}^{\prime}$ we have

$$
a(x)+\epsilon^{2} \Omega^{2}|x|^{2} \leq \frac{1}{2} a(x)<0 .
$$

Set

$$
U(x):=|u(x)|^{2} .
$$


We then calculate:

$$
\begin{aligned}
\frac{1}{2} \Delta U & =|\nabla u|^{2}+(u, \Delta u) \\
& =|\nabla u|^{2}+2 \Omega x^{\perp} \cdot(i u, \nabla u)-\frac{1}{\epsilon^{2}}(a(x)-U) U \\
& \geq-\Omega^{2}|x|^{2} U+\frac{1}{\epsilon^{2}}(U-a) U \\
& =\frac{1}{\epsilon^{2}} U\left(U-\left(a(x)+\epsilon^{2} \Omega^{2}|x|^{2}\right)\right) \\
& \geq \frac{1}{2 \epsilon^{2}}\left(a^{-}\right) U \geq 0
\end{aligned}
$$

where $a^{-}(x)=\max \{0,-a(x)\} \geq 0$, using $(2.7)$.

By the bound (2.4) and Cauchy-Schwartz,

$$
\int_{T} U=\int_{T}|u|^{2} \leq C \epsilon|\ln \epsilon|
$$

From the computation (2.8) we see that $U$ is subharmonic in $T_{\epsilon}^{\prime}$, and therefore for all $x$ such that $B_{\rho}(x) \subset T_{\epsilon}^{\prime}$ we have

$$
U(x) \leq \frac{1}{\pi \rho^{2}} \int_{B_{\rho}(x)} U \leq \frac{1}{\pi \rho^{2}} \int_{T} U \leq C \frac{\epsilon|\ln \epsilon|}{\rho^{2}}
$$

Taking $x \in T_{\epsilon}:=\left\{x \in T: \operatorname{dist}(x, \partial T)>\epsilon^{1 / 3}\right\}$ and $\rho=\epsilon^{1 / 3}$ we conclude

$$
U(x) \leq C \epsilon^{1 / 3}|\ln \epsilon|
$$

in particular $U(x) \rightarrow 0$ locally uniformly in $T$.

To complete the proof, we note that (2.8) also implies that $U$ is a subsolution of the equation $-\Delta w+\frac{a^{-}}{\epsilon^{2}} w=0$ in $T_{\epsilon}$. Since (for $\epsilon$ sufficiently small) $a^{-} \geq \epsilon^{2 / 3}$ in $T_{\epsilon}$ we then use the comparison principle (see Lemma 2 of $[\mathrm{BBH}]$ for example) to conclude

$$
|u(x)|^{2}=U(x) \leq C \epsilon^{1 / 3}|\ln \epsilon| \exp \left[\operatorname{dist}\left(x, \partial T_{\epsilon}\right) / 4 \epsilon^{2 / 3}\right],
$$

and the conclusion follows.

$\diamond$

\section{$2.3 \quad$ Splitting of the energy}

We apply the remarkable observation (see $[\mathrm{LM}]$ ) that the energy of the profile $\eta_{\epsilon}$ and the remaining complex order parameter $v=u / \eta_{\epsilon}$ decouple exactly into two independent pieces. 
Lemma 2.6 Let $u \in H_{0}^{1}(\mathcal{D})$. Then, $v=u / \eta_{\epsilon}$ is well defined, belongs to $H_{\eta_{\epsilon}^{2}}^{1}$ and

$$
\begin{gathered}
E_{\epsilon}(u)=J_{\epsilon}\left(\eta_{\epsilon}\right)+G_{\epsilon}(v) \text { where } G_{\epsilon}(v)=F_{\epsilon}(v)-\int_{\mathcal{D}} \eta_{\epsilon}^{2} \Omega \mathbf{x}^{\perp} \cdot(i v, \nabla v) d x \\
\text { and } F_{\epsilon}(v)=\int_{\mathcal{D}}\left\{\frac{\eta_{\epsilon}^{2}}{2}|\nabla v|^{2}+\frac{\eta_{\epsilon}^{4}}{4 \epsilon^{2}}\left(|v|^{2}-1\right)^{2}\right\} d x
\end{gathered}
$$

Proof: Note that $v$ is well defined in $\mathcal{D}$, since $\eta_{\epsilon}>0$. The decomposition and the fact that $v \in H_{\eta_{\epsilon}}^{1}(\mathcal{D})$ follow exactly as in [Se2]: since $\eta_{\epsilon}$ satisfies $(1.4)$, we multiply it by $\eta_{\epsilon}\left(1-|v|^{2}\right)$. This gives an identity that we use to compute $E_{\epsilon}(v)$.

\section{The giant vortex: velocity of order 1}

This is a case similar to the pinning case studied by [AnBaPh]. When $\Omega$ is fixed (independent of $\epsilon$ ) but sufficiently large, minimizers acquire non-zero degree. The solution $u$ does not vanish in the annular bulk of the condensate, since the vortices are pinned to the giant hole where $|u|$ is very small. The essential difference with $[\mathrm{AnBaPh}]$ is the fact that the hole here is large and $a$ is linear near the boundary: in $[\mathrm{AnBaPh}]$ the pinning sites are isolated zeros of the pinning potential $a$ and $\sqrt{a}$ is sufficiently regular to be used as a test function for the upper bound.

Before beginning the proof of Theorem 1.1, we need the following results.

Lemma 3.1 For $v \in H^{1}\left(\mathcal{A}, S^{1}\right)$, let

$$
G_{0}(v)=\int_{\mathcal{A}} \frac{a}{2}|\nabla v|^{2}-a \Omega \mathbf{x}^{\perp} \cdot(i v, \nabla v) .
$$

Then for any $r$ such that $\partial B_{r} \subset \mathcal{A}$,

$$
G_{0}(v) \geq g_{0}(d) \text { where } d=\operatorname{deg}\left(v, \partial B_{r}\right)
$$

and $g_{0}$ is given in (1.5). In particular, the minimum of $G_{0}$ is achieved, and any minimizer has the form $v_{0}=\alpha e^{i D_{0} \theta}$, where $D_{0}$ minimizes $g_{0}$ in $\mathbf{Z}$ and $\alpha \in \mathbf{C}$ is a constant with $|\alpha|=1$.

Proof: Let $v$ be a smooth function in $\mathcal{A}$ with values in $S^{1}$. The degree of $v$ is constant on any concentric circle $S_{r} \subset \mathcal{A}$; let us call it $d$. Define the energy of $v$ on the circle $S_{r}$ by

$$
e(r ; v)=\int_{S_{r}} \frac{a}{2}|\nabla v|^{2}-a \Omega \mathbf{x}^{\perp} \cdot(i v, \nabla v) .
$$


First, we note that

$$
\int_{S_{r}} a \Omega \mathbf{x}^{\perp} \cdot(i v, \nabla v)=2 \pi r a(r) \Omega d,
$$

depends only on the homotopy class of $v$. For the other term, the Cauchy-Schwartz inequality implies

$$
\int_{S_{r}}|\nabla v|^{2} \geq \int_{S_{r}}(i v, \nabla v)^{2} \geq \frac{1}{2 \pi r}\left(\int_{S_{r}}(i v, \nabla v) \cdot \tau\right)^{2}=\frac{2 \pi d^{2}}{r} .
$$

Therefore, we have the lower bound,

$$
e(r ; v) \geq 2 \pi a(r)\left(\frac{1}{2} \frac{d^{2}}{r}-\Omega d r\right)=e\left(r ; e^{i d \theta}\right)
$$

for any $v \in H^{1}\left(\mathcal{A}, S^{1}\right)$ and for almost every $r \in\left(r_{0}, R_{0}\right)$.

Now let $v$ be chosen to minimize $G_{0}$. Integrating (3.3) over $r$, we obtain

$$
g_{0}(d)=G_{0}\left(e^{i d \theta}\right) \leq G_{0}(v) \leq G_{0}\left(e^{i D_{0} \theta}\right)=g_{0}\left(D_{0}\right) .
$$

Since $D_{0}$ minimizes $g_{0}$ over $\mathbf{Z}$, we conclude $d=D_{0}$, and each of the inequalities above is actually an equality. Since $G_{0}(v)=\int_{r_{0}}^{R_{0}} e(r ; v) d r=G_{0}\left(e^{i D_{0} \theta}\right)$ and the integrands are pointwise bounded by (3.3), we conclude that equality must hold (almost everywhere) in (3.3), and therefore also in (3.2). The case of equality in Cauchy Schwarz inequality in the integrals over $S_{r}$ implies that $(i v, \nabla v) \cdot \tau=\alpha(r)$ (independent of $\theta$ ). Since the degree is independent of $S_{r}$, we have

$$
2 \pi \alpha(r) r=\int_{0}^{2 \pi}(i v, \nabla v) \cdot \tau r d \theta=2 \pi D_{0} .
$$

By the equality $|(i v, \nabla v) \cdot \tau|=|\nabla v|$ (and recalling $|v|=1$ ), we conclude that the normal derivative $(i v, \nabla v) \cdot n=0$ and integrating the relation $(i v, \nabla v) \cdot \tau=\frac{D_{0}}{r}$ gives $v=e^{i D\left(\theta-\theta_{0}\right)}$ with $\theta_{0} \in \mathbf{R}$ constant.

$\diamond$

We now present the proof of Theorem 1.1.

We first derive an upper bound on the energy of the minimizers. Let $\chi=\chi(r)$ be a smooth function in $\mathcal{D}, 0 \leq \chi(r) \leq 1$, with $\chi(r)=0$ for $0 \leq r \leq \frac{r_{0}}{4}$ and $\chi(r)=1$ for $\frac{r_{0}}{2} \leq r \leq R_{0}$. We use the test function $u=\eta_{\epsilon} v, v=\chi e^{i D_{0} \theta}$, where $D_{0} \in \mathbf{Z}$ minimizes $g_{0}(d)$. Then the splitting of the energy of Lemma 2.6 yields

$$
E_{\epsilon}(u)=J_{\epsilon}\left(\eta_{\epsilon}\right)+G_{\epsilon}\left(e^{i D_{0} \theta} ; \mathcal{A}\right)+G_{\epsilon}\left(\chi e^{i D_{0} \theta} ; T\right)
$$


Let us prove that the last term tends to 0 , using the exponential decay of $\eta_{\epsilon}$ in $B_{r_{0} / 2}$ Proposition $2.1(v)$ :

$$
\begin{aligned}
\int_{T} \eta_{\epsilon}^{2}\left|\nabla\left(\chi e^{i D_{0} \theta}\right)\right|^{2} & \leq 2 \int_{T}\left[\eta_{\epsilon}^{2} \chi^{2}\left|\nabla e^{i D_{0} \theta}\right|^{2}+\eta_{\epsilon}^{2}|\nabla \chi|^{2}\right] \\
& \leq C \int_{\frac{r_{0}}{4}}^{\frac{r_{0}}{2}} \eta_{\epsilon}^{2}\left[\frac{D_{0}^{2}}{r}+1\right] d r=o(1),
\end{aligned}
$$

and

$$
\frac{1}{\epsilon^{2}} \int_{T} \eta_{\epsilon}^{4}\left(1-\chi^{2}\right)^{2} \leq \frac{1}{\epsilon^{2}} \int_{\frac{r_{0}}{4}}^{\frac{r_{0}}{2}} \eta_{\epsilon}^{4} \leq \frac{C}{\epsilon^{2}} \exp \left(\frac{-r_{0}}{\epsilon^{2 / 3}}\right)=o(1)
$$

Note that $\lim _{\epsilon \rightarrow 0} G_{\epsilon}\left(e^{i D_{0} \theta} ; \mathcal{A}\right)=g_{0}\left(D_{0}\right)$, because of the convergence of $\eta_{\epsilon}^{2}$ to $a$. This implies

$$
E_{\epsilon}(u) \leq J_{\epsilon}\left(\eta_{\epsilon}\right)+g_{0}\left(D_{0}\right)+o(1)
$$

For the minimizer $u_{\epsilon}$, let $v_{\epsilon}=u_{\epsilon} / \eta_{\epsilon}$. We have $E_{\epsilon}\left(u_{\epsilon}\right)=J_{\epsilon}\left(\eta_{\epsilon}\right)+G_{\epsilon}\left(v_{\epsilon}\right)$. We get from (3.5)

$$
\limsup _{\epsilon \rightarrow 0} G_{\epsilon}\left(v_{\epsilon}\right) \leq g_{0}\left(D_{0}\right)
$$

Now we want to prove that $\liminf _{\epsilon \rightarrow 0} G_{\epsilon}\left(v_{\epsilon}\right) \geq g_{0}\left(D_{0}\right)$. We claim that for any $v \in H_{\eta_{\epsilon}^{2}}^{1}(\mathcal{D})$,

$$
\liminf _{\epsilon \rightarrow 0} G_{\epsilon}(v ; T) \geq 0
$$

Indeed, by the same basic steps as above,

$$
\begin{aligned}
\left|\int_{T} \Omega \eta_{\epsilon}^{2} x^{\perp} \cdot(i v, \nabla v)\right| & \leq \frac{1}{2} \int_{T} \eta_{\epsilon}^{2}|\nabla v|^{2}+\frac{\Omega^{2}}{2} \int_{T} \eta_{\epsilon}^{2}|x|^{2}|v|^{2} \\
& \leq \frac{1}{2} \int_{T} \eta_{\epsilon}^{2}|\nabla v|^{2}+\frac{\Omega^{2}}{2} \int_{T}\left[|x|^{2} \eta_{\epsilon}^{2}\left(|v|^{2}-1\right)+\eta_{\epsilon}^{2}|x|^{2}\right] \\
& \leq \frac{1}{2} \int_{T} \eta_{\epsilon}^{2}|\nabla v|^{2}+\frac{1}{4 \epsilon^{2}} \int_{T} \eta_{\epsilon}^{4}\left(|v|^{2}-1\right)^{2}+\frac{\Omega^{4} \epsilon^{2}}{4} \int_{T}|x|^{4}+\frac{\Omega^{2}}{2} \int_{T} \eta_{\epsilon}^{2}|x|^{2} \\
& =F_{\epsilon}(v)+o(1),
\end{aligned}
$$

and we therefore conclude, $G_{\epsilon}(v ; T) \geq o(1)$ as claimed.

Next we show some weak compactness of $v_{\epsilon}$ in the annulus to get the lower bound. We again use the same ideas to estimate the rotation term:

$$
\begin{aligned}
\left|\int_{\mathcal{A}} \eta_{\epsilon}^{2} x^{\perp} \cdot(i v, \nabla v)\right| & \leq \frac{1}{4} \int_{\mathcal{A}} \eta_{\epsilon}^{2}|\nabla v|^{2}+\frac{\Omega^{2}}{2} \int_{\mathcal{A}} \eta_{\epsilon}^{2}|x|^{2}|v|^{2} \\
& \leq \frac{1}{4} \int_{\mathcal{A}} \eta_{\epsilon}^{2}|\nabla v|^{2}+C \Omega^{2}\left[\int_{\mathcal{A}} \eta_{\epsilon}^{2}\left(|v|^{2}-1\right)+\int_{\mathcal{A}} \eta_{\epsilon}^{2}\right] \\
& \leq \frac{1}{4} \int_{\mathcal{A}} \eta_{\epsilon}^{2}|\nabla v|^{2}+\frac{1}{8 \epsilon^{2}} \int_{\mathcal{A}} \eta_{\epsilon}^{4}\left(|v|^{2}-1\right)^{2}+C \Omega^{2}\left[\int_{\mathcal{A}}|x|^{4}+\int_{\mathcal{A}} a+1\right] .
\end{aligned}
$$


Therefore,

$$
g_{0}\left(D_{0}\right)+o(1) \geq G_{\epsilon}\left(v_{\epsilon}\right) \geq G_{\epsilon}\left(v_{\epsilon} ; \mathcal{A}\right)+o(1) \geq \frac{1}{4} \int_{\mathcal{A}} \eta_{\epsilon}^{2}\left|\nabla v_{\epsilon}\right|^{2}+\frac{1}{8 \epsilon^{2}} \int_{\mathcal{A}} \eta_{\epsilon}^{4}\left(\left|v_{\epsilon}\right|^{2}-1^{2}\right)+O\left(\Omega^{2}\right) .
$$

As a consequence, there exists a constant $C$ (depending on $\Omega$ but independent of $\epsilon$ ) so that

$$
\int_{\mathcal{A}}\left(\eta_{\epsilon}^{2}|\nabla v|^{2}+\frac{1}{\epsilon^{2}} \eta_{\epsilon}^{4}\left(|v|^{2}-1\right)^{2}\right) \leq C
$$

In particular, along some subsequence we have $\eta_{\epsilon} \nabla v_{\epsilon} \rightarrow w_{0}$ weakly and $\left|v_{\epsilon}\right| \rightarrow 1$ strongly in $L^{2}(\mathcal{A})$. Fix any $\delta>0$ and let $\mathcal{A}_{\delta}:=\{x \in \mathcal{A}: \operatorname{dist}(x, \partial \mathcal{A})>\delta\}$. Then

$$
C \geq \int_{\mathcal{A}_{\delta}} \eta_{\epsilon}^{2}\left|\nabla v_{\epsilon}\right|^{2} \geq \frac{1}{2} \int_{\mathcal{A}_{\delta}} a\left|\nabla v_{\epsilon}\right|^{2}
$$

uniformly in $\epsilon$, using (iv) of Proposition 2.1. Hence, $\left(v_{\epsilon}\right)$ is bounded in $H^{1}\left(\mathcal{A}_{\delta}\right)$ for each $\delta>0$, and a further subsequence converges weakly in $H_{l o c}^{1}\left(\mathcal{A}_{\delta}\right)$, strongly in $L^{2}\left(\mathcal{A}_{\delta}\right)$ and pointwise almost everywhere. By a diagonal argument we obtain a limit function $v_{0}$, with $\left|v_{0}\right|=1$ and $v_{\epsilon} \rightarrow v_{0}$ in $\mathcal{A}_{\delta}$ for each $\delta>0$. By lower semicontinuity, for each $\delta>0$,

$$
\liminf _{\epsilon \rightarrow 0} \int_{\mathcal{A}} \eta_{\epsilon}^{2}\left|\nabla v_{\epsilon}\right|^{2} \geq \liminf _{\epsilon \rightarrow 0} \int_{\mathcal{A}_{\delta}} \eta_{\epsilon}^{2}\left|\nabla v_{\epsilon}\right|^{2} \geq \int_{\mathcal{A}_{\delta}} a\left|\nabla v_{0}\right|^{2}
$$

Letting $\delta \rightarrow 0$ we obtain $v_{0} \in H_{a}^{1}(\mathcal{A})$. By the pointwise convergence we may also identify the weak limit $w_{0}$ above: we have $\eta_{\epsilon} \nabla v_{\epsilon} \rightarrow \sqrt{a} \nabla v_{0}$ weakly in $L^{2}(\mathcal{A})$. We want to show that this convergence is in fact strong.

The rotation term also converges away from the boundary: by weak convergence of $\nabla v_{\epsilon}$, strong convergence of $v_{\epsilon}$, and uniform convergence of $\eta_{\epsilon}^{2}$ in $\mathcal{A}_{\delta}$, we have for each $\delta>0$,

$$
\Omega \int_{\mathcal{A}_{\delta}} \eta_{\epsilon}^{2} x^{\perp} \cdot\left(i v_{\epsilon}, \nabla v_{\epsilon}\right) \rightarrow \Omega \int_{\mathcal{A}_{\delta}} a x^{\perp} \cdot\left(i v_{0}, \nabla v_{0}\right)
$$

In particular, $\liminf _{\epsilon \rightarrow 0} G_{\epsilon}\left(v_{\epsilon} ; \mathcal{A}_{\delta}\right) \geq G_{0}\left(v_{0} ; \mathcal{A}_{\delta}\right)$. Let $\mathcal{N}_{\delta}:=\{x \in \mathcal{A}: \operatorname{dist}(x, \partial \mathcal{A}) \leq \delta\}$. By Lemma 3.1, (3.7), and the above, we have that for any $\gamma>0$ we may choose $\epsilon$ sufficiently small (in our subsequence) so that

$$
\begin{aligned}
\gamma+G_{0}\left(v_{0}\right) & \geq \gamma+G_{0}\left(e^{i D_{0} \theta}\right) \\
& \geq G_{\epsilon}\left(v_{\epsilon}\right) \\
& =G_{\epsilon}\left(v_{\epsilon} ; \mathcal{A}_{\delta}\right)+G_{\epsilon}\left(v_{\epsilon} ; \mathcal{N}_{\delta}\right)+G_{\epsilon}\left(v_{\epsilon} ; T\right) \\
& \geq G_{0}\left(v_{0} ; \mathcal{A}_{\delta}\right)+G_{\epsilon}\left(v_{\epsilon} ; \mathcal{N}_{\delta}\right)-\gamma
\end{aligned}
$$

and hence we may choose $\epsilon>0$ small enough so that

$$
G_{\epsilon}\left(v_{\epsilon} ; \mathcal{N}_{\delta}\right) \leq 2 \gamma+G_{0}\left(v_{0} ; \mathcal{N}_{\delta}\right) \leq 3 \gamma
$$


This allows us to estimate the rotation term in $\mathcal{N}_{\delta}$, for $\delta>0$ sufficiently small:

$$
\begin{aligned}
\left|\Omega \int_{\mathcal{N}_{\delta}} \eta_{\epsilon} \mathbf{x}^{\perp} \cdot\left(i v_{\epsilon}, \nabla v_{\epsilon}\right)\right| & \leq \frac{1}{4} \int_{\mathcal{N}_{\delta}} \eta_{\epsilon}^{2}\left|\nabla v_{\epsilon}\right|^{2}+C \Omega^{2} \int_{\mathcal{N}_{\delta}}\left(\eta_{\epsilon}^{2}\left(\left|v_{\epsilon}\right|^{2}-1\right)+\eta_{\epsilon}^{2}\right) \\
& \leq \frac{1}{4} \int_{\mathcal{N}_{\delta}} \eta_{\epsilon}^{2}\left|\nabla v_{\epsilon}\right|^{2}+C \Omega^{2}\left[\left(\int_{\mathcal{A}} \eta_{\epsilon}^{4}\left(\left|v_{\epsilon}\right|^{2}-1\right)^{2}\right)^{\frac{1}{2}}+\left(\max a^{+}\right)\left|\mathcal{N}_{\delta}\right|\right] \\
& \leq \frac{1}{4} \int_{\mathcal{N}_{\delta}} \eta_{\epsilon}^{2}\left|\nabla v_{\epsilon}\right|^{2}+\gamma \\
& \leq G_{\epsilon}\left(v_{\epsilon} ; \mathcal{N}_{\delta}\right)+\gamma \\
& \leq 4 \gamma
\end{aligned}
$$

for $\epsilon, \delta$ sufficiently small. Together with (3.9) we conclude that

$$
\lim _{\epsilon \rightarrow 0} \int_{\mathcal{A}} \eta_{\epsilon} \mathbf{x}^{\perp} \cdot\left(i v_{\epsilon}, \nabla v_{\epsilon}\right)=\int_{\mathcal{A}} a \mathbf{x}^{\perp} \cdot\left(i v_{0}, \nabla v_{0}\right)
$$

and from above

$$
\liminf _{\epsilon \rightarrow 0} G_{\epsilon}\left(v_{\epsilon}\right) \geq G_{0}\left(v_{0}\right) .
$$

Therefore $\lim _{\epsilon \rightarrow 0} G_{\epsilon}\left(v_{\epsilon}\right)=G_{0}\left(v_{0}\right)$ so that $\lim \int_{\mathcal{A}} \eta_{\epsilon}\left|\nabla v_{\epsilon}\right|^{2}=\int_{\mathcal{A}} a\left|\nabla v_{0}\right|^{2}$, and hence $\eta_{\epsilon} \nabla v_{\epsilon} \rightarrow$ $\sqrt{a} \nabla v_{0}$ strongly in $L^{2}(\mathcal{A})$, i.e. $v_{\epsilon} \rightarrow v_{0}$ strongly in $H_{l o c}^{1}(\mathcal{A})$, with $v_{0}$ a minimizer of $G_{0}$ in $H_{a}^{1}(\mathcal{A})$, that is $v_{0}=\alpha e^{i D_{0} \theta}$ with $|\alpha|=1$.

The uniform convergence of $\left|v_{\epsilon}\right| \rightarrow 1$ in $\mathcal{A}_{\delta}$ for any $\delta>0$, as well as the conclusion that $u \rightarrow 0$ in $H^{1}(T)$, follows from the same arguments as Step A.2 of the proof of Theorem 1 of $[\mathrm{BBH}]$, since the matching of the upper and lower bounds on $G_{\epsilon}\left(v_{\epsilon} ; \mathcal{A}\right)$ imply

$$
\frac{1}{\epsilon^{2}} \int_{\mathcal{A}} \eta_{\epsilon}^{4}\left(|v|^{2}-1\right)^{2}=o(1)
$$

This completes the proof of Theorem 1.1.

$\diamond$

\section{Vortices in the annulus and the critical velocity}

In this section, we consider larger rotation values of the type (1.8) and we expect to see the appearance of vortices in the annular bulk $\mathcal{A}=\left\{x: r_{0}<|x|<R_{0}\right\}$ of the condensate. We treat the model of the condensate restricted to the annulus $\mathcal{A}$, with Dirichlet condition $u \in H_{0}^{1}(\mathcal{A} ; \mathbf{C})$ on $\partial \mathcal{A}$ for simplicity, and denote by $E_{\epsilon}(u)=\hat{E}_{\epsilon}(u ; \mathcal{A})$ in the remainder of the paper. Similarly, we remove the hats for $\eta$. 


\subsection{Splitting the energy; vortex balls}

We refine our decomposition of Lemma 2.6 to incorporate the effect of the giant vortex. Define $v_{\epsilon}$ via

$$
u_{\epsilon}=\eta_{\epsilon} e^{i D_{\epsilon} \theta} v_{\epsilon}
$$

with

$$
D_{\epsilon}=\left[\frac{\Omega}{\Lambda_{1}}\right]
$$

where $[x]$ denotes the closest integer to $x$ and $\Lambda_{1}$ is given by (1.5). Recall that $\Omega=O(|\ln \epsilon|)$. Since $e^{i D_{\epsilon} \theta}$ is smooth and of modulus one in the annulus $\mathcal{A}$, by Lemma $2.6 v \in H_{\eta_{\epsilon}^{2}}^{1}(\mathcal{A})$ is well-defined, and (2.10) and a direct calculation yields:

$$
\begin{aligned}
E_{\epsilon}\left(u_{\epsilon}\right) & =E_{\epsilon}\left(\eta_{\epsilon}\right)+G_{\epsilon}\left(e^{i D_{\epsilon} \theta} v_{\epsilon}\right) \\
& =E_{\epsilon}\left(\eta_{\epsilon}\right)+\frac{1}{2} \Lambda_{\epsilon} D_{\epsilon}^{2}-\Omega M_{\epsilon} D_{\epsilon}+\mathcal{E}_{\epsilon}\left(v_{\epsilon}\right),
\end{aligned}
$$

with

$$
\begin{gathered}
\Lambda_{\epsilon}=\int_{\mathcal{A}} \frac{\eta_{\epsilon}^{2}}{|x|^{2}} \text { and } M_{\epsilon}=\int_{\mathcal{A}} \eta_{\epsilon}^{2}\left|v_{\epsilon}\right|^{2} \\
\mathcal{E}_{\epsilon}(v)=\int_{\mathcal{A}}\left\{\frac{\eta_{\epsilon}^{2}}{2}|\nabla v|^{2}-\eta_{\epsilon}^{2} \Omega \mathbf{X} \cdot(i v, \nabla v)+\frac{\eta_{\epsilon}^{4}}{4 \epsilon^{2}}\left(|v|^{2}-1\right)^{2}\right\}
\end{gathered}
$$

and $\mathbf{X}=x^{\perp}-\frac{D_{\epsilon}}{\Omega} \nabla \theta$.

Using $\eta_{\epsilon} e^{i D_{\epsilon} \theta}$ as a test function for an upper bound, we find that if $u_{\epsilon}$ is a minimizer, then $\mathcal{E}_{\epsilon}\left(v_{\epsilon}\right) \leq 0$. Our aim is to compute a lower bound and thus locate the vortices.

Our first step is to excise a thin neighborhood of the two edges where $a(r)$ vanishes. We do this since our energy method is not sensitive enough to discern vortices which are very close to the edges of the condensate, where the density $|u|^{2} \sim a$ is already very small. Let

$$
\delta=\delta_{\epsilon}=\frac{(\ln |\ln \epsilon|)^{1 / 4}}{|\ln \epsilon|}
$$

and

$$
\mathcal{A}_{\delta_{\epsilon}}:=\left\{x \in \mathcal{A}: \operatorname{dist}(x, \partial \mathcal{A})>\delta_{\epsilon}\right\}, \quad \mathcal{N}_{\epsilon}:=\mathcal{A} \backslash \mathcal{A}_{\delta_{\epsilon}} .
$$

Then, by familiar arguments we have:

$$
\begin{aligned}
& \Omega \int_{\mathcal{N}_{\epsilon}} \eta_{\epsilon}^{2} \mathbf{X} \cdot(i v, \nabla v) \leq \frac{1}{2} \int_{\mathcal{N}_{\epsilon}} \eta_{\epsilon}^{2}|\nabla v|^{2}+C \Omega^{2} \int_{\mathcal{N}_{\epsilon}}|v|^{2} \eta_{\epsilon}^{2} \\
& \quad=\frac{1}{2} \int_{\mathcal{N}_{\epsilon}} \eta_{\epsilon}^{2}|\nabla v|^{2}+\Omega^{2} C \int_{\mathcal{N}_{\epsilon}}\left[\eta_{\epsilon}^{2}\left(|v|^{2}-1\right)+\left(\eta_{\epsilon}^{2}-a(r)\right)+a(r)\right] \\
& \quad \leq \frac{1}{2} \int_{\mathcal{N}_{\epsilon}} \eta_{\epsilon}^{2}|\nabla v|^{2}+\int_{\mathcal{N}_{\epsilon}} \frac{\eta_{\epsilon}^{4}}{8 \epsilon^{2}}\left(|v|^{2}-1\right)^{2}+C \Omega^{2}\left(\left|\mathcal{N}_{\epsilon}\right| \epsilon^{2}+\epsilon \sqrt{J_{\epsilon}\left(\eta_{\epsilon}\right)\left|\mathcal{N}_{\epsilon}\right|}+C \delta_{\epsilon}\left|\mathcal{N}_{\epsilon}\right|\right) \\
& \quad \leq \frac{1}{2} \int_{\mathcal{N}_{\epsilon}} \eta_{\epsilon}^{2}|\nabla v|^{2}+\int_{\mathcal{N}_{\epsilon}} \frac{\eta_{\epsilon}^{4}}{8 \epsilon^{2}}\left(|v|^{2}-1\right)^{2}+C \sqrt{\ln |\ln \epsilon|} .
\end{aligned}
$$


In particular,

$$
\mathcal{E}_{\epsilon}\left(v ; \mathcal{N}_{\epsilon}\right) \geq-C \sqrt{\ln |\ln \epsilon|}
$$

and consequently,

$$
\mathcal{E}_{\epsilon}\left(v ; \mathcal{A}_{\delta_{\epsilon}}\right) \leq C \sqrt{\ln |\ln \epsilon|}
$$

for any minimizer.

Note that by the same steps as in (4.5) above,

$$
\Omega \int_{\mathcal{A}_{\delta_{\epsilon}}} \eta_{\epsilon}^{2} \mathbf{X} \cdot(i v, \nabla v) \leq \frac{1}{4} \int_{\mathcal{A}_{\delta_{\epsilon}}} \eta_{\epsilon}^{2}|\nabla v|^{2}+\frac{1}{8 \epsilon^{2}} \int_{\mathcal{A}_{\delta_{\epsilon}}} \eta_{\epsilon}^{4}\left(|v|^{2}-1\right)^{2}+C \Omega^{2}
$$

and hence from (4.6) we obtain the useful estimate

$$
\int_{\mathcal{A}_{\delta_{\epsilon}}}\left\{\eta_{\epsilon}^{2}|\nabla v|^{2}+\frac{\eta_{\epsilon}^{4}}{\epsilon^{2}}\left(|v|^{2}-1\right)^{2}\right\} \leq C \Omega^{2}=O\left(|\ln \epsilon|^{2}\right)
$$

with $C$ independent of $\epsilon$.

We now define

$$
\tilde{\mathcal{E}}_{\epsilon}(v):=\int_{\mathcal{A}_{\delta_{\epsilon}}}\left\{\frac{a}{2}|\nabla v|^{2}-\Omega a \mathbf{X} \cdot(i v, \nabla v)+\frac{a^{2}}{4 \epsilon^{2}}\left(|v|^{2}-1\right)^{2}\right\},
$$

and using the estimates on $\left(\eta_{\epsilon}^{2}-a\right)$ in $\mathcal{A}_{\epsilon}$ from Proposition 2.1 (iv) and (4.7), (4.8), we conclude:

$$
\tilde{\mathcal{E}}_{\epsilon}(v) \leq \mathcal{E}_{\epsilon}\left(v ; \mathcal{A}_{\delta_{\epsilon}}\right)\left(1+o\left(\epsilon^{1 / 3}|\ln \epsilon|\right)\right) \leq c \sqrt{\ln |\ln \epsilon|}
$$

Moreover, the bounds (4.7), (4.8) also hold with a replacing $\eta_{\epsilon}^{2}$,

$$
\int_{\mathcal{A}_{\delta_{\epsilon}}}\left\{a|\nabla v|^{2}+\frac{a^{2}}{\epsilon^{2}}\left(|v|^{2}-1\right)^{2}\right\},\left|\Omega \int_{\mathcal{A}_{\delta_{\epsilon}}} a \mathbf{X} \cdot(i v, \nabla v)\right| \leq C \Omega^{2}=O\left(|\ln \epsilon|^{2}\right) .
$$

Now in $\mathcal{A}_{\delta_{\epsilon}}$, we may isolate the vortices using the method of Sandier [Sa] and Sandier \& Serfaty [SSe3]. We have the following result:

Proposition 4.1 For any $C>0$ there exist positive constants $\epsilon_{0}, C_{0}$ so that for any $\epsilon<\epsilon_{0}$, $\Omega \leq C|\ln \epsilon|$ and any $v$ with $\tilde{\mathcal{E}}_{\epsilon}(v) \leq C[\ln |\ln \epsilon|]^{1 / 2}$ there exists a finite collection $\left\{B_{i}=\right.$ $\left.B\left(p_{i}, s_{i}\right)\right\}_{i=1, \ldots, m}$ of disjoint balls such that:

$$
\begin{gathered}
\left\{x \in \mathcal{A}_{\delta_{\epsilon}}:|v|<1-|\ln \epsilon|^{-4}\right\} \subset \bigcup_{i=1}^{m} B_{i} ; \\
\sum_{i=1}^{m} s_{i}<|\ln \epsilon|^{-8} ; \\
\operatorname{deg}_{\partial B_{i}}\left(\frac{v}{|v|}\right):=d_{i} \quad \text { for all } i \\
\int_{B_{i}} \frac{a}{2}|(\nabla-i \Omega \mathbf{X}) v|^{2} \geq \pi a\left(p_{i}\right)\left|d_{i}\right|\left(|\ln \epsilon|-C_{0} \ln |\ln \epsilon|\right) \quad \text { for all } i .
\end{gathered}
$$


We sketch the proof, as the details are minor modifications of the analogous results in [SSe1], [SSe3]. First, we complete the square in the gradient term and, using (4.11) obtain:

$$
\int_{\mathcal{A}_{\delta_{\epsilon}}}\left(\frac{a}{2}|(\nabla-i \Omega \mathbf{X}) v|^{2}+\frac{a^{2}}{4 \epsilon^{2}}\left(|v|^{2}-1\right)^{2}\right) \leq \tilde{\mathcal{E}}_{\epsilon}(v)+4 \Omega^{2} \int_{\mathcal{A}_{\delta_{\epsilon}}} \eta_{\epsilon}^{2}|X|^{2}|v|^{2}+o(1) \leq C|\ln \epsilon|^{2} .
$$

Since $a(x) \geq C \delta_{\epsilon}$ for $x \in \mathcal{A}_{\delta_{\epsilon}}$, setting $\rho:=|v|$ we have

$$
\delta_{\epsilon}^{2} \int_{\mathcal{A}_{\delta_{\epsilon}}}\left(\frac{1}{2}|\nabla \rho|^{2}+\frac{1}{4 \epsilon^{2}}\left(\rho^{2}-1\right)^{2}\right) \leq C|\ln \epsilon|^{2},
$$

and hence

$$
\int_{\mathcal{A}_{\delta_{\epsilon}}}\left(\frac{1}{2}|\nabla \rho|^{2}+\frac{1}{4 \epsilon^{2}}\left(\rho^{2}-1\right)^{2}\right) \leq C|\ln \epsilon|^{4} .
$$

Let $\mathcal{A}_{\delta_{\epsilon}, t}:=\left\{x \in \mathcal{A}_{\delta_{\epsilon}}: \rho<1-t\right\}$, and $\gamma_{t}=\partial \mathcal{A}_{\delta_{\epsilon}, t}$. Using the co-area formula as in [SSe3], there exists $t_{0} \in\left(0,|\ln \epsilon|^{-4}\right)$ and a finite set of balls $B_{1}, \ldots, B_{k}$ with radii $s_{1}, \ldots, s_{k}$ which cover $\gamma_{t_{0}}$, satisfying $\sum_{i} s_{i} \leq C \epsilon|\ln \epsilon|^{8}$. In $\mathcal{A}_{\delta_{\epsilon}} \backslash \mathcal{A}_{\delta_{\epsilon}, t_{0}}$ we may write $v=\rho e^{i \phi}$ for a (possibly multi-valued) $H_{l o c}^{1}$ function $\phi(x)$.

We then let the balls grow continuously, using the process described in [Sa], [SSe3], to obtain a lower bound in the expanding balls,

$$
\int_{B_{i} \backslash \mathcal{A}_{\delta_{\epsilon}, t_{0}}} \frac{a}{2}|\nabla \phi-\Omega \mathbf{X}|^{2} \geq \pi\left(\min _{B_{i}} a\right)\left|d_{i}\right|\left(\ln \epsilon-\bar{C}_{0} \ln |\ln \epsilon|\right),
$$

with constant $\bar{C}_{0}$ independent of $\epsilon$. Note that the minimum of $a(x)$ over $B_{i}$ is non-increasing as the radii increase and as balls are merged (when they touch in the expansion process.) We terminate the process when the sum of the radii of the balls equals $|\ln \epsilon|^{-8}$. By continuity of $a(x)$ we may then replace the minimum of $a$ on each ball by the value at its center $p_{i}$, making an error which is small compared to $a\left(p_{i}\right)$ itself. This error can then be absorbed into the coefficient of $\ln |\ln \epsilon|$.

Finally,

$$
\begin{aligned}
\int_{B_{i}} \frac{a}{2}|(\nabla-i \Omega \mathbf{X}) v|^{2} & \geq \int_{B_{j} \backslash \mathcal{A}_{\delta_{\epsilon}, t_{0}}} \frac{a}{2}\left(1+\rho^{2}-1\right)|\nabla \phi-\Omega \mathbf{X}|^{2} \\
& \geq\left(1-C|\ln \epsilon|^{-4}\right) \int_{B_{j} \backslash A_{\epsilon, t_{0}}} \frac{a}{2}|\nabla \phi-\Omega \mathbf{X}|^{2} \\
& \geq\left(1-C|\ln \epsilon|^{-4}\right)\left(\pi a\left(p_{i}\right)\left|d_{i}\right|\left(|\ln \epsilon|-\bar{C}_{0} \ln |\ln \epsilon|\right)\right) \\
& \geq \pi a\left(p_{i}\right)\left|d_{i}\right|\left(|\ln \epsilon|-C_{0} \ln |\ln \epsilon|\right),
\end{aligned}
$$

for constant $C_{0}$ independent of $\epsilon$, which completes the proof of the Proposition. 


\subsection{The potential function}

In this section, we define a potential function which will enter into our expansion of the energy and in the end completely determine the location of the vortices. For $\delta>0$ and $\theta \in \mathbf{R}$, let us define

$$
F_{\theta, \delta}(r):=\int_{r}^{R_{0}-\delta} a(s)\left(s-\left(\Lambda_{1}^{-1}-\theta\right) \frac{1}{s}\right) d s
$$

Here we take $\delta=\delta_{\epsilon}$ as in the previous section in (4.4), and

$$
\theta=\theta_{\epsilon}=\frac{1}{\Lambda_{1}}-\frac{D_{\epsilon}}{\Omega}=\left(\frac{\Omega}{\Lambda_{1}}-\left[\frac{\Omega}{\Lambda_{1}}\right]\right) \frac{1}{\Omega}
$$

so that

$$
\left|\theta_{\epsilon}\right| \leq \frac{1}{\Omega}=O\left(|\ln \epsilon|^{-1}\right)
$$

$F_{\theta_{\epsilon}, \delta_{\epsilon}}$ enters into our problem because it is the primative of the vector field $a(r) X$ which vanishes at the outer edge of $\mathcal{A}_{\delta_{\epsilon}}$,

$$
\nabla^{\perp}\left(F_{\theta_{\epsilon}, \delta_{\epsilon}}(r)\right)=a(r) X, \quad F_{\theta, \delta}\left(R_{0}-\delta\right)=0
$$

for any $\delta_{\epsilon}, \theta_{\epsilon}$. It will be important later on to notice that

$$
F_{\theta, \delta}^{\prime}\left(r_{0}\right)=0=F_{\theta, \delta}^{\prime}\left(R_{0}\right)
$$

and when $\theta=0=\delta$,

$$
F_{0,0}\left(r_{0}\right)=0=F_{0,0}\left(R_{0}\right), \quad F_{0,0}(r)>0 \text { for all } r \in\left(r_{0}, R_{0}\right) .
$$

Lemma 4.2 Suppose $a(r)$ is real analytic, $a(r)>0$ for $r \in\left(r_{0}, R_{0}\right)$. Then there exists a finite collection $\Gamma=\left\{r_{1}^{*}, \ldots, r_{m}^{*}\right\} \subset\left(r_{0}, R_{0}\right)$ such that

$$
\max _{r \in\left[r_{0}, R_{0}\right]} \frac{F_{0,0}(r)}{a(r)}=: K_{0}
$$

is attained for each $r^{*} \in \Gamma$. Moreover, there exist constants $K_{1}>0$ and $M \geq 1$ such that

$$
\frac{F_{0,0}(r)}{a(r)} \leq K_{0}-\frac{K_{1}}{\sqrt{|\ln \epsilon|}}
$$

whenever $\operatorname{dist}(r, \Gamma) \geq|\ln \epsilon|^{-1 / 2 M}$. 
Proof: Set

$$
k_{0,0}(r):=\frac{F_{0,0}(r)}{a(r)} .
$$

Then $k_{0,0}$ extends to a real analytic function on $\left[r_{0}, R_{0}\right]$ with $k_{0,0}\left(r_{0}\right)=0=k_{0,0}\left(R_{0}\right)$ and $k_{0,0}(r)>0$ in $\left(r_{0}, R_{0}\right)$. The maximum is therefore attained in the interior, and on a finite collection of points, which we call $\Gamma$. At each maximum point the degree of degeneracy is finite, and hence

$$
k_{0,0}(r) \sim K_{0}-c_{j}\left(r-r_{j}^{*}\right)^{2 N_{j}}
$$

in some neighborhood $I_{j}$ of each $r_{j}^{*} \in \Gamma$, with $N_{j} \in \mathbf{N}$. In the complement $r \in\left[r_{0}, R_{0}\right] \backslash \cup I_{j}$, $k_{0,0}(r)<K_{0}$ so by compactness we can find $\beta>0$ (independent of $\epsilon$ ) such that

$$
k_{0,0}(r)<K_{0}-\beta \quad \text { for all } r \in\left[r_{0}, R_{0}\right] \backslash \cup I_{j} .
$$

Together with (4.23) in each $I_{j}$ we obtain (4.22).

$\diamond$

Lemma 4.3 There exist constants $K_{2}, K_{3}>0$ such that

$$
\frac{\left|F_{\theta_{\epsilon}, \delta_{\epsilon}}(r)\right|}{a(r)} \leq K_{0}+\frac{K_{2}}{|\ln \epsilon|}
$$

whenever $r \in\left(r_{0}+\delta_{\epsilon}, R_{0}-\delta_{\epsilon}\right)$, and

$$
\frac{\left|F_{\theta_{\epsilon}, \delta_{\epsilon}}(r)\right|}{a(r)} \leq K_{0}-\frac{K_{3}}{\sqrt{|\ln \epsilon|}}
$$

when $\operatorname{dist}(r, \Gamma) \geq|\ln \epsilon|^{-1 / 2 M}$, where $K_{0}, M$ are as in Lemma 4.2.

Proof: We have

$$
\left|F_{\theta_{\epsilon}, \delta_{\epsilon}}(r)-F_{0,0}(r)\right| \leq C\left(\left|\theta_{\epsilon}\right|+\delta_{\epsilon}^{2}\right)
$$

with constant $C$ independent of $\epsilon$. Let $\alpha<\frac{1}{2} \min \left\{\alpha_{0}, \beta_{0}\right\}$. Then for any $\gamma>0$ sufficiently small, there exists a constant $a_{0}>0$ so that

$$
a(r) \geq \begin{cases}\alpha\left(r-r_{0}\right), & \text { if } r_{0}<r<r_{0}+\gamma \\ a_{0}, & \text { if } r_{0}+\gamma \leq r \leq R_{0}-\gamma \\ \alpha\left(R_{0}-r\right), & \text { if } R_{0}-\gamma<r<R_{0}\end{cases}
$$

When $r_{0}+\delta_{\epsilon}<r<r_{0}+\gamma$, we calculate:

$$
\begin{aligned}
\left|F_{\theta_{\epsilon}, \delta_{\epsilon}}(r)\right| & \leq\left|F_{0,0}(r)\right|+C\left(\left|\theta_{\epsilon}\right|+\delta_{\epsilon}{ }^{2}\right) \\
& \leq C\left(r-r_{0}\right)^{2}+C\left(\left|\theta_{\epsilon}\right|+\delta_{\epsilon}{ }^{2}\right)
\end{aligned}
$$


using (4.18), (4.19), and (4.20). Therefore,

$$
\begin{aligned}
\frac{\left|F_{\theta_{\epsilon}, \delta_{\epsilon}}(r)\right|}{a(r)} & \leq \frac{C}{\alpha} \gamma+\frac{C}{\alpha}\left(\frac{\left|\theta_{\epsilon}\right|+\delta_{\epsilon}{ }^{2}}{\delta_{\epsilon}}\right) \\
& \leq C \gamma+O\left([\ln |\ln \epsilon|]^{-1 / 4}\right) \\
& <\frac{1}{2} K_{0},
\end{aligned}
$$

by fixing a value of $\gamma$ sufficiently small. An analogous estimate holds on the interval $\left[R_{0}-\right.$ $\left.\gamma, R_{0}-\delta_{\epsilon}\right]$.

It remains to consider the larger interval, $r_{0}+\gamma \leq r \leq R_{0}-\gamma$. By choosing $\gamma$ smaller if necessary we may be sure that $\Gamma$ lies completely in this interval. Since

$$
\left|\frac{F_{\theta_{\epsilon}, \delta_{\epsilon}}(r)}{a(r)}-\frac{F_{0,0}(r)}{a(r)}\right| \leq \frac{C}{a_{0}}\left(\left|\theta_{\epsilon}\right|+\delta_{\epsilon}{ }^{2}\right) \leq C|\ln \epsilon|^{-1},
$$

the conclusion follows from Lemma 4.2.

$\diamond$

\subsection{A lower bound expansion}

We define $\mathcal{C}$ to be the set of concentric circles of radii $r^{*} \in \Gamma$, with $\Gamma$ as in Lemma 4.2.

The proof of the Theorem 1.2 is based on a detailed lower bound expansion of the energy in terms of the location and degrees of the vortex balls $\left(B_{i}\right)$ constructed in Proposition 4.1. First, we consider the energy in the balls themselves. We have

$$
\begin{aligned}
\int_{B_{i}} \frac{a}{2}\left(|\nabla v|^{2}-2 \Omega \mathbf{X} \cdot(i v, \nabla v)\right) & =\int_{B_{i}} \frac{a}{2}\left(|(\nabla-i \Omega \mathbf{X}) v|^{2}-\Omega^{2}|X|^{2}|v|^{2}\right) \\
& \geq \pi a\left(p_{i}\right)\left|d_{i}\right|\left(|\ln \epsilon|-C_{0} \ln |\ln \epsilon|\right)-o(1)
\end{aligned}
$$

from Proposition 4.1, where we have estimated the extra term using (4.13). We may also evaluate the cross-term in the region $A_{\epsilon} \backslash \cup B_{i}$ in terms of the potential functions $F_{\theta_{\epsilon}, \delta_{\epsilon}}$ introduced in the previous paragraph. First note that by slightly modifying our choice of $\delta_{\epsilon}$ we may be sure that no vortex ball intersects the inner or outer boundaries $\partial B_{r_{0}+\delta_{\epsilon}}(0)$, $\partial B_{R_{0}-\delta_{\epsilon}}(0)$ of the annulus $\mathcal{A}_{\delta_{\epsilon}}$. Indeed, if this is not the case by (4.13) we may find a constant $k_{\epsilon} \in[1,2)$ so that replacing $\delta_{\epsilon}{ }^{\prime}=k_{\epsilon} \delta_{\epsilon}$ we may avoid vortex balls intersecting the boundary.

Lemma 4.4 Let $d_{0}=\operatorname{deg}\left(v /|v| ; \partial B_{r_{0}+\delta_{\epsilon}}\right)$. Then,

$$
\Omega \int_{\mathcal{A}_{\delta_{\epsilon}} \backslash \cup B_{i}} a(x) \mathbf{X} \cdot(i v, \nabla v)=-2 \pi d_{0} F_{\theta_{\epsilon}, \delta_{\epsilon}}\left(r_{0}+\delta_{\epsilon}\right) \Omega+\sum_{i} 2 \pi d_{i} F_{\theta_{\epsilon}, \delta_{\epsilon}}\left(\left|p_{i}\right|\right) \Omega+o(1) .
$$


Proof: $\quad$ In $\mathcal{A}_{\delta_{\epsilon}} \backslash \cup B_{i}$ we may define $w=\frac{v}{|v|}$. Then $(i v, \nabla v)=|v|^{2}(i w, \nabla w)$, and

$$
\begin{aligned}
\left|\Omega \int_{\mathcal{A}_{\delta_{\epsilon}} \backslash \cup B_{i}} a \mathbf{X} \cdot(i v, \nabla v)-\Omega \int_{\mathcal{A}_{\delta_{\epsilon}} \backslash \cup B_{i}} a \mathbf{X} \cdot(i w, \nabla w)\right| & =\left|\Omega \int_{\mathcal{A}_{\delta_{\epsilon} \backslash \cup B_{i}}} a\left(|v|^{2}-1\right) \mathbf{X} \cdot(i w, \nabla w)\right| \\
& \leq C \Omega\left(\int_{\mathcal{A}_{\delta_{\epsilon}}} a^{2}\left(|v|^{2}-1\right)^{2}\right)^{1 / 2}\|\nabla w\|_{2} \\
& \leq C \epsilon|\ln \epsilon|^{2}\left(\frac{1}{\min _{\mathcal{A}_{\delta_{\epsilon}}} a} \int_{\mathcal{A}_{\delta_{\epsilon}}} a|\nabla v|^{2}\right)^{1 / 2} \\
& \leq C \frac{\epsilon|\ln \epsilon|^{3}}{\sqrt{\delta_{\epsilon}}},
\end{aligned}
$$

where we have used the basic energy estimates (4.11), hypothesis (H2) (on the vanishing of $a$ near $\partial \mathcal{A})$ and the observation that $|\nabla v| \geq|v||\nabla w| \geq\left(1-|\ln \epsilon|^{-5}\right)|\nabla w| \geq \frac{1}{2}|\nabla w|$ in $\mathcal{A}_{\delta_{\epsilon}} \backslash \cup B_{i}$. Since $|w|=1,(i w, \nabla w)$ is locally a gradient and is irrotational. Introducing the potential $F_{\theta_{\epsilon}, \delta_{\epsilon}}$ from the previous section,

$$
a(x) \mathbf{X}=\nabla^{\perp} F_{\theta_{\epsilon}, \delta_{\epsilon}}(|x|),
$$

and applying Stokes' Theorem we obtain:

$$
\begin{aligned}
\int_{\mathcal{A}_{\delta_{\epsilon}} \backslash \cup B_{i}} a \mathbf{X} \cdot(i w, \nabla w)= & \int_{\mathcal{A}_{\delta_{\epsilon}} \backslash \cup B_{i}} \nabla\left(F_{\theta_{\epsilon}, \delta_{\epsilon}}(|x|)\right) \cdot\left(i w, \nabla^{\perp} w\right) \\
= & -\int_{\partial B_{R_{0}-\delta_{\epsilon}}} F_{\theta_{\epsilon}, \delta_{\epsilon}}\left(R_{0}-\delta_{\epsilon}\right)\left(i w, \partial_{\tau} w\right) \\
& \quad+\int_{\partial B_{r_{0}+\delta_{\epsilon}}} F_{\theta_{\epsilon}, \delta_{\epsilon}}\left(r_{0}+\delta_{\epsilon}\right)\left(i w, \partial_{\tau} w\right) \\
& +\sum_{i} \int_{\partial B_{i}} F_{\theta_{\epsilon}, \delta_{\epsilon}}(|x|)\left(i w, \partial_{\tau} w\right) \\
= & -2 \pi d_{0} F_{\theta_{\epsilon}, \delta_{\epsilon}}\left(r_{0}+\delta_{\epsilon}\right)+\sum_{i} \int_{\partial B_{i}} F_{\theta_{\epsilon}, \delta_{\epsilon}}(|x|)\left(i w, \partial_{\tau} w\right) .
\end{aligned}
$$

Note that we have used (4.18) to eliminate one boundary term, and $d_{0}=\operatorname{deg}\left(w, \partial \mathcal{A}_{\delta_{\epsilon}}\right)$ to evaluate the other. The summation is over vortex balls in $\mathcal{A}_{\delta_{\epsilon}}$, as defined in Proposition 4.1.

To conclude we claim that for each vortex ball,

$$
\left|\Omega \int_{\partial B_{i}}\left(F_{\theta_{\epsilon}, \delta_{\epsilon}}(|x|)-F_{\theta_{\epsilon}, \delta_{\epsilon}}\left(\left|p_{i}\right|\right)\right)\left(i w, \partial_{\tau} w\right)\right| \leq \frac{|\ln \epsilon|^{3}}{\delta_{\epsilon}{ }^{2}} s_{i},
$$

where we recall that $s_{i}$ is the radius of $B_{i}$. This conclusion follows step-by-step from Lemma II.3 of [SSe1], where we replace their $h_{e x} \xi_{0}$ by our $\Omega F_{\theta_{\epsilon}, \delta_{\epsilon}}$. Note that $\left|\nabla F_{\theta_{\epsilon}, \delta_{\epsilon}}\right|$ is uniformly bounded independent of $\epsilon$, and $\|\nabla w\|_{2}$ is bounded in terms of the energy using 
the same trick as in (4.30) above.

$\diamond$

Putting (4.28) and (4.29) together we obtain the lower bound,

$$
\begin{aligned}
O(\sqrt{\ln |\ln \epsilon|}) \geq & \tilde{\mathcal{E}}_{\epsilon}(v) \\
\geq & \pi \sum a\left(p_{i}\right)\left|d_{i}\right|\left(|\ln \epsilon|-C_{0} \ln |\ln \epsilon|\right)-2 \pi \sum d_{i} F_{\theta_{\epsilon}, \delta_{\epsilon}}\left(\left|p_{i}\right|\right) \Omega \\
& \quad-2 \pi d_{0} F_{\theta_{\epsilon}, \delta_{\epsilon}}\left(r_{0}+\delta_{\epsilon}\right) \Omega+\frac{1}{2} \int_{\mathcal{A}_{\delta_{\epsilon}} \backslash \cup B_{i}} a|\nabla v|^{2}+o(1) .
\end{aligned}
$$

The behavior of $a(r)$ and $F_{0,0}(r)$ allow us to choose $\gamma>0$ (independent of $\epsilon$ ) such that

$$
\begin{gathered}
r_{0}+\gamma<\inf \Gamma \leq \sup \Gamma<R_{0}-\gamma, \\
a(r) \geq a_{0}:=\min \left\{a\left(r_{0}+\gamma\right), a\left(R_{0}-\gamma\right)\right\} \quad \text { for all } r \in\left[r_{0}+\gamma, R_{0}-\gamma\right],
\end{gathered}
$$

and

$$
\frac{F_{0,0}(r)}{a(r)}<\frac{K_{0}}{3} \quad \text { for all } r \in\left(r_{0}, r_{0}+\gamma\right) \cup\left(R_{0}-\gamma, R_{0}\right)
$$

Let

$$
\begin{aligned}
Z_{\gamma} & :=\left\{i: \delta_{\epsilon}<\operatorname{dist}\left(p_{i}, \partial \mathcal{A}\right) \leq \gamma,\right. \\
Z_{*} & :=\left\{i: \operatorname{dist}\left(p_{i}, \Gamma\right)<|\ln \epsilon|^{-1 / 2 M} \text { and } d_{i} \geq 0\right\}, \\
Z_{-} & :=\left\{i: \operatorname{dist}\left(p_{i}, \Gamma\right)<|\ln \epsilon|^{-1 / 2 M} \text { and } d_{i} \leq 0\right\}, \\
Z_{0} & :=\left(Z_{*} \cup Z_{-} \cup Z_{\gamma}\right)^{C},
\end{aligned}
$$

and set

$$
N_{x}:=\sum_{Z_{x}} a\left(p_{i}\right)\left|d_{i}\right|, \quad x=\gamma, *,-, 0 ; \quad \hat{N}=\sum a\left(p_{i}\right)\left|d_{i}\right|=N_{\gamma}+N_{*}+N_{-}+N_{0} .
$$

For vortices $p_{i}$ with $i \in Z_{\gamma}$, we use (4.27) and (4.32) to derive

$$
\frac{\left|F_{\theta_{\epsilon}, \delta_{\epsilon}}\left(\left|p_{i}\right|\right)\right|}{a\left(p_{i}\right)}<\frac{K_{0}}{2}
$$

Lemma 4.3 and (4.31) then yield

$$
\begin{aligned}
C \sqrt{\ln |\ln \epsilon|} \geq \pi \hat{N} & \left(|\ln \epsilon|-C_{0} \ln |\ln \epsilon|\right) \\
& -2 \pi K_{0} N_{*}\left[1+O\left(|\ln \epsilon|^{-1}\right)\right]\left(\omega_{0}|\ln \epsilon|+\omega_{1} \ln |\ln \epsilon|\right) \\
& +2 \pi K_{0} N_{-}\left[1+O\left(|\ln \epsilon|^{-1}\right)\right]\left(\omega_{0}|\ln \epsilon|+\omega_{1} \ln |\ln \epsilon|\right) \\
& -2 \pi K_{0} N_{\gamma}\left[\frac{1}{2}+O\left(|\ln \epsilon|^{-1}\right)\right]\left(\omega_{0}|\ln \epsilon|+\omega_{1} \ln |\ln \epsilon|\right) \\
& -2 \pi K_{0} N_{0}\left[1-\frac{K_{4}}{\sqrt{|\ln \epsilon|}}\right]\left(\omega_{0}|\ln \epsilon|+\omega_{1} \ln |\ln \epsilon|\right) \\
& -2 \pi d_{0} F_{\theta_{\epsilon}, \delta_{\epsilon}}\left(r_{0}+\delta_{\epsilon}\right) \Omega+\int_{\mathcal{A}_{\delta_{\epsilon}} \backslash \cup B_{i}} \frac{a}{2}|\nabla v|^{2}+o(1) .
\end{aligned}
$$


One difficulty in dealing with this lower bound expansion is the boundary term at $r_{0}+\delta_{\epsilon}$, since we have no a priori bound on the degree $d_{0}$ of the inner edge of the annulus. We must consider two cases separately.

Case I: $\left|d_{0}\right| \leq 2 \sum\left|d_{i}\right|$.

Recalling (4.19), (4.20) and the behavior of $a(r)$ near $r=r_{0}$, we have

$$
0<\frac{F_{0,0}\left(r_{0}+\delta_{\epsilon}\right)}{a\left(r_{0}+\delta_{\epsilon}\right)} \leq C \delta_{\epsilon} .
$$

With (4.26) we obtain

$$
\begin{aligned}
\left|F_{\theta_{\epsilon}, \delta_{\epsilon}}\left(r_{0}+\delta_{\epsilon}\right)\right| & \leq C \delta_{\epsilon} a\left(r_{0}+\delta_{\epsilon}\right)+C\left(\left|\theta_{\epsilon}\right|+\delta_{\epsilon}^{2}\right) \\
& \leq C\left(\delta_{\epsilon}+\frac{\left|\theta_{\epsilon}\right|}{a\left(r_{0}+\delta_{\epsilon}\right)}\right) a\left(r_{0}+\delta_{\epsilon}\right) \\
& \leq \frac{C}{[\ln |\ln \epsilon|]^{1 / 4}} a\left(r_{0}+\delta_{\epsilon}\right) .
\end{aligned}
$$

Hence,

$$
\left|d_{0} \Omega F_{\theta_{\epsilon}, \delta_{\epsilon}}\left(r_{0}+\delta_{\epsilon}\right)\right| \leq \frac{C}{[\ln |\ln \epsilon|]^{1 / 4}} a\left(r_{0}+\delta_{\epsilon}\right)|\ln \epsilon| \sum\left|d_{i}\right|
$$

Hence

$$
\begin{aligned}
2 \pi\left|d_{0} \Omega F_{\theta_{\epsilon}, \delta_{\epsilon}}\left(r_{0}+\delta_{\epsilon}\right)\right| & \leq \frac{C}{[\ln |\ln \epsilon|]^{1 / 4}} a\left(r_{0}+\delta_{\epsilon}\right)|\ln \epsilon|\left[\sum_{Z_{\gamma}}\left|d_{i}\right|+\sum_{Z_{\gamma}^{C}}\left|d_{i}\right|\right] \\
& \leq \frac{C|\ln \epsilon|}{[\ln |\ln \epsilon|]^{1 / 4}} \sum_{Z_{\gamma}} a\left(p_{i}\right)\left|d_{i}\right|+\frac{C \delta_{\epsilon}}{[\ln |\ln \epsilon|]^{1 / 4}} \sum_{Z_{\gamma}^{C}} \frac{a\left(p_{i}\right)}{a_{0}}\left|d_{i}\right| \\
& \leq \frac{\pi}{8}|\ln \epsilon| \sum_{Z_{\gamma}} a\left(p_{i}\right)\left|d_{i}\right|+\frac{C}{|\ln \epsilon|} \sum_{Z_{\gamma}^{C}} a\left(p_{i}\right)\left|d_{i}\right| .
\end{aligned}
$$

We now substitute back into the lower bound for the energy (4.34):

$$
\begin{aligned}
C \sqrt{\ln |\ln \epsilon|} \geq & \pi\left(N_{*}+N_{0}\right)|\ln \epsilon|\left(1-2 K_{0} \omega_{0}\right) \\
& +\pi N_{-}|\ln \epsilon|\left(1+2 K_{0} \omega_{0}\right)(1+o(1))+\pi N_{\gamma}|\ln \epsilon|\left(\frac{7}{8}-K_{0} \omega_{0}\right)(1+o(1)) \\
& -\pi N_{*}\left(C_{0}+2 K_{0} \omega_{1}\right) \ln |\ln \epsilon|+C N_{0} \sqrt{|\ln \epsilon|}+\int_{\mathcal{A}_{\delta_{\epsilon}} \backslash \cup B_{i}} \frac{a}{2}|\nabla v|^{2}+o(1 \mid 4.36)
\end{aligned}
$$

In this step we have used Lemma 4.3, (4.33), and the choice of the angular speed (1.8).

Our first step is to conclude that the minimizers exhibit no vortices in the bulk when the speed is too small. Let $\omega_{0}^{*}=1 / 2 K_{0}$. From (4.36), we derive

$$
\omega_{0}<\omega_{0}^{*} \text {, then } \hat{N}=\sum a\left(p_{i}\right)\left|d_{i}\right| \leq C \frac{\sqrt{\ln |\ln \epsilon|}}{|\ln \epsilon|} .
$$


Suppose $\omega_{0}=\omega_{0}^{*}$, and

$$
\omega_{1}<-\frac{C_{0}}{2 K_{0}} .
$$

Then each term on the right-hand side of (4.36) is non-negative, and we conclude

$$
\hat{N}=\sum a\left(p_{i}\right)\left|d_{i}\right| \leq C[\ln |\ln \epsilon|]^{-1 / 2} .
$$

Because of the weight $a(x)$ we cannot conclude that the total degree of vortices in $\mathcal{A}_{\delta_{\epsilon}}$ is zero, but we can make that conclusion if we restrict our attention to a smaller domain. Let $\rho=\rho_{\epsilon}>>(\ln |\ln \epsilon|)^{-1 / 2} ;$ for example, $\rho_{\epsilon}=[\ln |\ln \epsilon|]^{-1 / q}$ with $q<\frac{1}{2}$ will do. Then

$$
\rho_{\epsilon} \sum_{\operatorname{dist}\left(p_{i}, \partial \mathcal{A}\right)>\rho_{\epsilon}}\left|d_{i}\right| \leq \hat{N} \leq C[\ln |\ln \epsilon|]^{-1 / 2}
$$

which implies

$$
\sum_{\operatorname{dist}\left(p_{i}, \partial \mathcal{A}\right)>\rho_{\epsilon}}\left|d_{i}\right|=o(1)
$$

that is we expect not to see any vortices at any distance larger than $[\ln |\ln \epsilon|]^{-1 / 2}$ from $\partial \mathcal{A}$ when the rotation is slower than this critical value.

When the angular speed is larger,

$$
\omega_{1}>-\frac{C_{0}}{2 K_{0}}
$$

we rearrange the terms in $(4.36)$ to arrive at

$$
\left(N_{0}+N_{-}+N_{\gamma}\right) \sqrt{|\ln \epsilon|} \leq C N_{*} \ln |\ln \epsilon|+C \sqrt{\ln |\ln \epsilon|}
$$

with $C$ independent of $\epsilon$. We are going to bound $N_{*}$ and therefore infer that the essential contribution to the weighted sum of vortices in the bulk is due to positive degree vortices concentrating at the minimal set $\Gamma$. To complete the argument we must use the remaining term in the energy. Denote by $I_{\epsilon}:=\left(r_{0}+\delta_{\epsilon}, R_{0}-\delta_{\epsilon}\right)$. By Proposition 4.1 the set

$$
J_{\epsilon}:=\left\{r \in I_{\epsilon}: \partial B_{r}(0) \cap \bigcup B_{s_{i}}\left(p_{i}\right)=\emptyset\right\}
$$

is a finite union of intervals whose complement $\left|I_{\epsilon} \backslash J_{\epsilon}\right|<|\ln \epsilon|^{-12}$ has very small measure. For each $r \in J_{\epsilon}|v| \geq 1-|\ln \epsilon|^{-4}$ and hence we may define

$$
D(r):=\operatorname{deg}\left(\frac{v}{|v|}, \partial B_{r}(0)\right) .
$$

Let $r_{1}:=r_{0}+\gamma, r_{2}:=R_{0}-\gamma$, and fix any $t_{1}, t_{2}$ with

$$
r_{1}<t_{1}<\inf \Gamma \leq \sup \Gamma<t_{2}<r_{2} .
$$


Note that $r_{1}, r_{2}, t_{1}, t_{2}$ are all independent of $\epsilon$. In $\left(r_{1}, r_{2}\right)$, we recall that $a(r) \geq a_{0}>0$.

On the one hand,

$$
\left|D\left(t_{1}\right)-D\left(r_{1}\right)\right|=\left|\sum_{t_{1}<\left|p_{i}\right|<r_{1}} d_{i}\right| \leq \sum_{t_{1}<\left|p_{i}\right|<r_{1}} \frac{a\left(p_{i}\right)}{a_{0}}\left|d_{i}\right| \leq \frac{N_{0}}{a_{0}} \leq o(1) N_{*} .
$$

In the same way we show that $\left|D\left(r_{2}\right)-D\left(t_{2}\right)\right| \leq o(1) N_{*}$. Finally,

$$
\begin{aligned}
\left|D\left(t_{2}\right)-D\left(t_{1}\right)\right| & =\left|\sum_{\substack{t_{1}<\left|p_{i}\right|<t_{2}\\
}} d_{i}\right| \\
& \geq \sum_{\substack{t_{1}<\left|p_{i}\right|<t_{2} \\
d_{i} \geq 0}} d_{i}-\sum_{\substack{t_{1}<\left|p_{i}\right|<t_{2} \\
d_{i}<0}} d_{i} \\
& \geq \frac{1}{\bar{a}} N_{*}-C\left(N_{-}+N_{0}\right) \\
& \geq \frac{1}{\bar{a}} N_{*}(1-o(1))
\end{aligned}
$$

In particular, it follows that

$$
\min \left\{\left|D\left(t_{1}\right)\right|,\left|D\left(t_{2}\right)\right|\right\} \geq \frac{1}{2 \bar{a}} N_{*} .
$$

Suppose that it's $\left|D\left(t_{1}\right)\right| \geq \frac{1}{2 \bar{a}} N_{*}$. Then we have $|D(r)| \geq \frac{1}{4 \bar{a}} N_{*}$ for every $r \in\left[r_{1}, t_{1}\right]$. Writing $v=|v| e^{i \phi}$ (for $|x|=r \in J_{\epsilon}$ ) we estimate the remainins term in the energy as follows, using that in $J_{\epsilon},|v| \geq 1-|\ln \epsilon|^{-4}$ :

$$
\begin{aligned}
& \int_{\mathcal{A}_{\delta_{\epsilon} \backslash \cup B_{i}} \frac{1}{2}|\nabla v|^{2}} \geq \int_{J_{\epsilon}} \int_{0}^{2 \pi} \frac{a(r)}{2}|v|^{2}|\nabla \phi|^{2} r d \theta d r \\
& \geq \int_{J_{\epsilon}} \int_{0}^{2 \pi} \frac{a(r)}{2}|\nabla \phi|^{2} r d \theta d r(1+o(1)) \\
& \geq \pi \int_{J_{\epsilon}} \frac{a(r)}{r}(D(r))^{2}(1+o(1)) \\
& \geq C N_{*}^{2}(1+o(1)) .
\end{aligned}
$$

Returning to the estimate (4.36) we see

$$
C \sqrt{\ln |\ln \epsilon|} \geq C_{1} N_{*}^{2}-C_{2} N_{*} \ln |\ln \epsilon|+o(1),
$$

with constants $C_{1}, C_{2}$ independent of $\epsilon$. We conclude that

$$
N_{*} \leq C \ln |\ln \epsilon| .
$$

With (4.38) and (4.40) we have

$$
\max \left\{N_{-}, N_{0}, N_{\gamma}\right\} \leq C \frac{(\ln |\ln \epsilon|)^{3 / 2}}{|\ln \epsilon|^{1 / 2}} .
$$


As before, we need to further restrict the domain in order to come to a conclusion as to the total degree in the annulus. Take any $\rho_{\epsilon}$ with

$$
\rho_{\epsilon}>>\frac{(\ln |\ln \epsilon|)^{3 / 2}}{|\ln \epsilon|^{1 / 2}}
$$

and then

$$
\sum_{\substack{\operatorname{dist}\left(p_{i}, \Gamma\right)>|\ln \epsilon|-1 / 2 M \\ \operatorname{dist}\left(p_{i}, \partial \mathcal{A}\right)>\rho_{\epsilon}}}\left|d_{i}\right|+\sum_{\substack{d_{i}<0 \\ \operatorname{dist}\left(p_{i}, \partial \mathcal{A}\right)>\rho_{\epsilon}}}\left|d_{i}\right| \leq C \frac{(\ln |\ln \epsilon|)^{3 / 2}}{|\ln \epsilon|^{1 / 2} a\left(\rho_{\epsilon}\right)} \rightarrow 0 .
$$

Since the left-hand side is now an integer, it must be exactly zero for $\epsilon$ sufficiently small.

Finally, we consider the degree of the neighborhood of the edge of the annulus. From the previous paragraph we observe that $D(r) \equiv D_{1}$ is constant in the interval $r \in\left[r_{0}+\rho_{\epsilon}, t_{1}\right]$. We return to the lower bound (4.36) to obtain

$$
\begin{aligned}
C \sqrt{\ln |\ln \epsilon|}+C N_{*} \ln |\ln \epsilon| & \geq \int_{\mathcal{A}_{\delta_{\epsilon}} \backslash \cup B_{i}} \frac{a}{2}|\nabla v|^{2} \\
& \geq \pi\left|\int_{r_{1}}^{s_{1}} \frac{a}{r}(D(r))^{2} d r\right|+o(1) \\
& \geq C D_{1}^{2},
\end{aligned}
$$

In particular, given the bound (4.40) we have

$$
\left|\operatorname{deg}\left(\frac{v}{|v|}, \partial B_{r_{0}+\rho_{\epsilon}}(0)\right)\right|=\left|D_{1}\right| \leq C \ln |\ln \epsilon| .
$$

Note that this confirms that we have made a good choice of the degree $D_{\epsilon}$ of the giant vortex, since for the original wave function $u$ we have

$$
\operatorname{deg}\left(\frac{u}{|u|}, \partial B_{r_{0}+\rho_{\epsilon}}(0)\right)=D_{\epsilon}+O(\ln |\ln \epsilon|) .
$$

This concludes the analysis for Case I.

Case II: $\left|d_{0}\right|>2 \sum\left|d_{i}\right|$.

Let $D(r), J_{\epsilon}$ be as in the previous part, so

$$
|D(r)|=\left|d_{0}+\sum_{\left|p_{i}\right| \leq r} d_{i}\right| \geq \frac{1}{2}\left|d_{0}\right| \quad \text { for all } r \in J_{\epsilon} .
$$

We then estimate as before,

$$
\begin{aligned}
\int_{\mathcal{A}_{\delta_{\epsilon} \backslash \cup B_{i}}} \frac{a}{2}|\nabla v|^{2} & \geq \int_{J_{\epsilon}} \int_{0}^{2 \pi} \frac{a}{2}|v|^{2}|\nabla \phi|^{2} \\
& \geq \pi \int_{J_{\epsilon}} \frac{a}{r}(D(r))^{2} d r+o(1) \\
& \geq \frac{\pi}{2} d_{0}^{2} \int_{J_{\epsilon}} \frac{a}{r} d r=C_{1} d_{0}^{2} .
\end{aligned}
$$


On the other hand, in Case II, using the estimate for $F_{\theta_{\epsilon}, \delta_{\epsilon}}$

$$
\left|2 \pi d_{0} \Omega F_{\theta_{\epsilon}, \delta_{\epsilon}}\left(r_{0}+\delta_{\epsilon}\right)\right| \leq \frac{C}{(\ln |\ln \epsilon|)^{1 / 4}} d_{0} \delta_{\epsilon}|\ln \epsilon| \leq C_{2}\left|d_{0}\right|
$$

so from (4.31) we get:

$$
\begin{aligned}
C \sqrt{\ln |\ln \epsilon|} \geq & \tilde{\mathcal{E}}_{\epsilon}(v) \\
& \geq C_{1} d_{0}^{2}-C_{2} d_{0}+\pi \sum a\left(p_{i}\right)\left|d_{i}\right|\left(|\ln \epsilon|-C_{0} \ln |\ln \epsilon|\right) \\
& \quad-2 \pi \sum d_{i} F_{\theta_{\epsilon}, \delta_{\epsilon}}\left(\left|p_{i}\right|\right) \Omega+o(1) .
\end{aligned}
$$

We may now repeat the same steps as in Case I (although we no longer need to distinguish $\left.Z_{\gamma}, N_{\gamma}\right)$ to derive:

$$
C \sqrt{\ln |\ln \epsilon|} \geq C_{1} d_{0}^{2}-C_{2}\left|d_{0}\right|+C_{3}\left(N_{0}+N_{-}\right) \sqrt{|\ln \epsilon|}-C N_{*} \ln |\ln \epsilon|
$$

hence

$$
\left(N_{-}+N_{0}\right) \leq C \sqrt{\frac{\ln |\ln \epsilon|}{|\ln \epsilon|}}+C\left|d_{0}\right| \frac{\ln |\ln \epsilon|}{\sqrt{|\ln \epsilon|}}
$$

This leads again to

$$
C_{1} d_{0}^{2}-C\left|d_{0}\right| \ln |\ln \epsilon| \leq C \sqrt{\ln |\ln \epsilon|}
$$

and thus the same conclusions as in Case I. This completes the proof of parts (i) and (ii) of Theorem 1.2.

\subsection{Upper bounds on the energy}

In order to prove the lower bounds on the number of vortices claimed in Theorem 1.2 we need to refine our upper bound on the energy for the case $\omega_{0}=\omega_{0}^{*}$ and $\omega_{1}>-\omega_{1}^{*}$. We do this in two ways, the first which will guarantee that there is at least one essential vortex provided $\omega_{1}>0$, and the second which will give the lower bound claimed in part (iii) of Theorem 1.2, $\mathbf{D}_{\epsilon} \geq c \ln |\ln \epsilon|$.

First, assume $\omega_{1}>0$. We will construct a test function of the form $u=\eta_{\epsilon} e^{i D_{\epsilon} \theta} v_{p_{0}}$, where $v_{p_{0}}$ has a vortex on $\mathcal{C}: v_{p_{0}}(x)=f_{\epsilon}\left(\left|x-p_{0}\right|\right) \frac{x-p_{0}}{\left|x-p_{0}\right|}$, where $\left|p_{0}\right| \in \Gamma, f_{\epsilon}(0)=0$ and $f_{\epsilon}(\hat{R})=1$. If we fix $\hat{R}>0$ so that $\mathcal{D} \supset B_{\hat{R}}\left(p_{0}\right)$ we then have:

$$
\begin{aligned}
\tilde{\mathcal{E}}_{\epsilon}\left(v_{p}\right) & \leq \int_{B_{\hat{R}}\left(p_{0}\right)}\left(\frac{a}{2}\left|\nabla f_{\epsilon}\right|^{2}+\frac{a^{2}}{4 \epsilon^{2}}\left(f_{\epsilon}^{2}-1\right)^{2}+\frac{a}{r^{2}} f_{\epsilon}^{2}\right)-2 \pi \Omega F_{\theta_{\epsilon}, \delta_{\epsilon}}\left(p_{0}\right)+o(1) \\
& \leq \pi a\left(p_{0}\right)|\ln \epsilon|-2 \pi\left(\frac{1}{2 K_{0}}|\ln \epsilon|+\omega_{1} \ln |\ln \epsilon|\right)\left(K_{0} a\left(p_{0}\right)+O\left(|\ln \epsilon|^{-1}\right)\right)+C \\
& \leq-2 \pi K_{0} a\left(p_{0}\right) \omega_{1} \ln |\ln \epsilon|+C .
\end{aligned}
$$


We now return to our lower bound from (4.36), or the analogous inequality in Case II. We now know that $N_{0}, N_{-}, N_{\gamma}=o(1)$, and therefore with this improved upper bound we obtain

$$
-\pi N_{*}\left(C_{0}+2 K_{0} \omega_{1}\right) \leq-2 \pi K_{0} a\left(p_{0}\right) \omega_{1}+o(1) .
$$

This can be rewritten in the form of a lower bound for $N_{*}$,

$$
N_{*} \geq \frac{2 K_{0} a(\Gamma) \omega_{1}}{C_{0}+2 K_{0} \omega_{1}}+o(1),
$$

and hence for $\omega_{1}>0$ sufficiently large we must have at least one essential vortex.

Finally, we derive an upper bound with $n_{\epsilon}=[\ln |\ln \epsilon|]$ vortices regularly placed on a circle $C=\partial B_{r_{*}}(0)$ in $\mathcal{C}$. Indeed, we believe that the minimizers should strongly resemble this configuration when the rotation is near the critical value. The construction uses the following modified Green's functions which give a kind of harmonic conjugate function for solutions with vortices in $\mathcal{A}$.

To avoid some technical difficulties associated with the vanishing of $a$ at $\partial \mathcal{A}$ we fix $\gamma$ with $0<\gamma<a\left(r_{*}\right)$ and consider the truncation

$$
a_{\gamma}(r):=\max \{a(r), \gamma\}
$$

In particular, $a_{\gamma}(r)=a(r)$ in a neighborhood of the curve $C$. Following [SSe3, Se2, ASSe] we use a Green's function to construct our trial function.

Lemma 4.5 For every fixed $y \in \mathcal{A}$ there exists $G(x, y)$ in $W^{1, q}$ for any $q<2$ and constant $\alpha>0$ such that

$$
\begin{aligned}
-\operatorname{div}_{x}\left(\frac{1}{a_{\gamma}} \nabla_{x} G(x, y)\right) & =\delta_{y}(x) \quad \text { in } \mathcal{D}^{\prime}(\mathcal{A}) \\
\left.G(x, y)\right|_{x \in \partial B_{R_{0}}} & =0 \\
\left.G(x, y)\right|_{x \in \partial B_{r_{0}}} & =\alpha \\
\int_{\partial B_{r_{0}}} \frac{1}{a_{\gamma}} \frac{\partial G}{\partial \nu_{x}} & =0 .
\end{aligned}
$$

Moreover:

(i) $G(x, y) \geq 0$ in $x \in \overline{\mathcal{A}} \backslash\{y\}$ for each $y \in \mathcal{A}$.

(ii) For any compact set $K \subset \subset \mathcal{A}$ there exists a constant $C_{K}$ such that

$$
\left|G(x, y)+\frac{a_{\gamma}(x)}{2 \pi} \ln \right| x-y|| \leq C(K)
$$

for all $y \in K$ and $x \in \overline{\mathcal{A}}$. 
(iii) For any $y \in \mathcal{A}, G(x, y)$ is continuous for $x \in \overline{\mathcal{A}} \backslash\{y\}$.

Proof: Let $\tilde{G}(x, y)$ denote the usual Dirichlet Green's function (satisfying (4.42), (4.43), and (4.44) with constant zero.) The existence of such a Green's function is standard, since the linear operator is uniformly elliptic in $\mathcal{A}$. The desired function is then a simple modification of $\tilde{G}$. First, if the pole $y$ is not contained in $\overline{B_{t}(0)} \backslash B_{s}(0)$, the divergence theorem insures that

$$
\int_{\partial B_{t}(0)} \frac{1}{a_{\gamma}} \frac{\partial \tilde{G}}{\partial r}-\int_{\partial B_{s}(0)} \frac{1}{a_{\gamma}} \frac{\partial \tilde{G}}{\partial r}=0 .
$$

In particular, $\int_{\partial B_{t}(0)} \frac{1}{a_{\gamma}} \frac{\partial \tilde{G}}{\partial r}$ is constant for all $t$ near $r_{0}$ and therefore $\int_{\partial B_{r_{0}}(0)} \frac{1}{a_{\gamma}} \frac{\partial \tilde{G}}{\partial \nu_{x}}$ is welldefined provided $y$ remains away from $\partial \mathcal{A}$. Let $\xi$ be the unique solution of

$$
\operatorname{div}\left(\frac{1}{a_{\gamma}} \nabla \xi\right)=0 \text { in } \mathcal{A}, \quad \xi=0 \text { on } \partial B_{R_{0}}, \quad \xi=1 \text { on } \partial B_{r_{0}} .
$$

This solution is obtained explicitly as

$$
\xi(r)=\lambda \int_{r}^{R_{0}} \frac{a_{\gamma}(s)}{s} d s, \quad \lambda=\left(\int_{r_{0}}^{R_{0}} \frac{a_{\gamma}(s)}{s} d s\right)^{-1},
$$

and satisfies

$$
\int_{\partial B_{r_{0}}} \frac{1}{a_{\gamma}} \frac{\partial \xi}{\partial \nu}=-\int_{\partial B_{r_{0}}} \frac{1}{a_{\gamma}} \frac{\partial \xi}{\partial r}=\frac{2 \pi \lambda}{r_{0}}>0
$$

a positive constant. Since $\frac{\partial \tilde{G}}{\partial \nu_{x}}=-\frac{\partial \tilde{G}}{\partial r} \leq 0$ on $\partial B_{r_{0}}(0)$ we may choose a constant $\alpha>0$ so that $G(x, y)+\alpha \xi(|x|)$ satisfies (4.45).

$\diamond$

To construct the upper bound, we adapt a construction from [SSe3]. (See also [Se2], [ASSe]). The difference is that instead of taking a alttice of vortices, we put them on a circle. Choose a radius $r_{*} \in \Gamma$ (defined as the set of minimizing radii in Lemma 4.2,) and let $C$ be the circle of radius $r_{*}$ centered at the origin. Choose $n_{\epsilon}=[\ln |\ln \epsilon|]$ points $\left\{p_{i}\right\}_{i=1, \ldots, n_{\epsilon}}$ which are equidistributed on $C$. In particular, we have $\left|p_{i}-p_{j}\right| \geq C / n_{\epsilon}$ for some $C>0$. Define

$$
f_{\epsilon}^{i}(x)=\frac{2}{\epsilon^{2}} \chi_{B_{\epsilon}\left(p_{i}\right)}
$$

the characteristic function of the ball $B_{\epsilon}\left(p_{i}\right)$, normalized to have total integral $2 \pi$. Let

$$
f_{\epsilon}(x)=\frac{1}{n_{\epsilon}} \sum_{i=1}^{n_{\epsilon}} f_{\epsilon}^{i}(x)
$$

The $f_{\epsilon}$ converges in the sense of measures, $f_{\epsilon} \rightarrow \mu_{*}=\frac{1}{r_{*}} \delta_{C}$, the arclength measure on $C$ normalized to have mass $2 \pi$. 
We define a "conjugate function" $h_{\epsilon}(x)$ as

$$
h_{\epsilon}(x)=n_{\epsilon} \int_{\mathcal{A}} G(x, y) f_{\epsilon}(y) d y \text {. }
$$

As $f_{\epsilon} \in L^{\infty}(\mathcal{A})$ we have $h_{\epsilon}$ is a weak solution of

$$
\begin{aligned}
-\operatorname{div}\left(\frac{1}{a_{\gamma}} \nabla h_{\epsilon}\right) & =n_{\epsilon} f_{\epsilon} \\
\left.h\right|_{\partial B_{R_{0}}} & =0 \\
\left.h\right|_{\partial B_{r_{0}}} & =\text { constant } \\
\int_{\partial B_{r_{0}}} \frac{1}{a_{\gamma}} \frac{\partial h_{\epsilon}}{\partial \nu} & =0 .
\end{aligned}
$$

Moreover, integration by parts and a straight-forward estimate using (4.46) shows that for any fixed $\epsilon>0$,

$$
\int_{\mathcal{A}} \frac{1}{a_{\gamma}}|\nabla h|^{2} d x=n_{\epsilon}^{2} \int_{\mathcal{A}} \int_{\mathcal{A}} G(x, y) f_{\epsilon}(x) f_{\epsilon}(y) d x d y<\infty
$$

and the quantization of the $f_{\epsilon}$ implies that

$$
\int_{\partial B_{R_{0}}} \frac{1}{a} \frac{\partial h_{\epsilon}}{\partial \nu}=2 \pi n_{\epsilon}
$$

We next use $h_{\epsilon}$ to define a test function $V$ for $\mathcal{E}_{\epsilon}$. First, define the phase $\phi(x)$ in $\mathcal{A} \backslash \cup B_{\epsilon}\left(p_{i}\right)$ by

$$
\nabla \phi(x)=\frac{1}{a_{\gamma}} \nabla^{\perp} h_{\epsilon}(x) .
$$

Note that $\phi$ is well-defined locally, as $\frac{1}{a_{\gamma}} \nabla^{\perp} h_{\epsilon}$ is irrotational in $\mathcal{A} \backslash \cup B_{\epsilon}\left(p_{i}\right)$ by the equation solved by $h_{\epsilon}$. Moreover, the quantization of the source terms $f_{\epsilon}^{i}$ ensures that $\phi$ defines a single-valued function modulo $2 \pi$, since for any closed curve $\sigma$ in $\mathcal{A} \backslash \cup B_{\epsilon}\left(p_{i}\right)$ we have

$$
\int_{\sigma} \nabla \phi \cdot \tau d s=\int_{\sigma} \frac{1}{a_{\gamma}} \nabla h_{\epsilon} \cdot \nu d s=2 \pi N_{\sigma}
$$

for some $N_{\sigma} \in \mathbf{Z}$, whether $\sigma$ winds around any of the holes or not. Take a smooth function $\rho_{0}(x)$ such that $0 \leq \rho_{0}(x) \leq 1, \rho_{0}(x) \equiv 1$ in $|x| \geq 2$, and $\rho_{0}(x) \equiv 0$ when $|x| \leq 1$. Define

$$
\rho(x)=\rho_{\epsilon}(x)= \begin{cases}\rho_{0}\left(\frac{x-p_{i}}{\epsilon}\right), & \text { when } x \in B_{2 \epsilon}\left(p_{i}\right), i=1, \ldots, n_{\epsilon}, \\ 1, & \text { when } x \in \mathcal{A} \backslash \cup B_{2 \epsilon}\left(p_{i}\right) .\end{cases}
$$

Note that

$$
\int_{\mathcal{A}}\left(a|\nabla \rho|^{2}+\frac{a^{2}}{2 \epsilon^{2}}\left(\rho^{2}-1\right)^{2}\right) \leq C n_{\epsilon}
$$


with constant $C$ independent of $\epsilon$. Then define

$$
V(x):=\rho(x) e^{i \phi(x)}
$$

where we interpret this to mean that $V(x) \equiv 0$ in $\cup B_{\epsilon}\left(p_{i}\right)$.

We now calculate the energy $\mathcal{E}_{\epsilon}(V)$. First,

$$
\begin{aligned}
\int_{\mathcal{A}}\left(a|\nabla V|^{2}+\frac{a^{2}}{2 \epsilon^{2}}\left(|V|^{2}-1\right)^{2}\right) & =\int_{\mathcal{A} \backslash \cup B_{\epsilon}\left(p_{i}\right)}\left(a|\nabla \rho|^{2}+\frac{a^{2}}{2 \epsilon^{2}}\left(\rho^{2}-1\right)^{2}+a \rho^{2}|\nabla \phi|^{2}\right) \\
& \leq \int_{\mathcal{A} \backslash \cup B_{\epsilon}\left(p_{i}\right)} a_{\gamma}|\nabla \phi|^{2}+O\left(n_{\epsilon}\right) \\
& \leq \int_{\mathcal{A}} \frac{1}{a_{\gamma}}\left|\nabla h_{\epsilon}\right|^{2}+O\left(n_{\epsilon}\right) \\
& =n_{\epsilon}^{2} \int_{\mathcal{A}} \int_{\mathcal{A}} G(x, y) f_{\epsilon}(x) f_{\epsilon}(y) d x d y+O\left(n_{\epsilon}\right) \\
& =\sum_{i, j=1}^{n_{\epsilon}} \int_{\mathcal{A}} \int_{\mathcal{A}} G(x, y) f_{\epsilon}^{i}(x) f_{\epsilon}^{j}(y) d x d y+O\left(n_{\epsilon}\right), \quad
\end{aligned}
$$

which is finite by the remark (4.49) above. To estimate this term we separate the domain $\mathcal{A}$ into two pieces. Let $\alpha>0$ be given, and set $\Delta_{\alpha}:=\{x, y:|x-y|<\alpha\}$. Since $G(x, y)$ is continuous on $\mathcal{A} \times \mathcal{A} \backslash \Delta_{\alpha}$, and $f_{\epsilon}(x) d x \rightarrow d \mu_{*}(x)$ weakly in meaures on $\mathcal{A}$ we have:

$$
\begin{aligned}
\lim _{\epsilon \rightarrow 0} \iint_{(\mathcal{A} \times \mathcal{A}) \backslash \Delta_{\alpha}} G(x, y) f_{\epsilon}(x) f_{\epsilon}(y) d x d y & =\iint_{\mathcal{A} \times \mathcal{A}} G(x, y) d \mu_{*}(x) d \mu_{*}(y) \\
& =\iint_{C \times C} G(x, y) d \mu_{*}(x) d \mu_{*}(y)=: A_{*} .
\end{aligned}
$$

Notice that the last term in (4.51) is finite, since by the estimate (4.46) we have $G(x, y) \in$ $L^{1}\left(C \times C, d \mu_{*} \otimes d \mu_{*}\right)$.

Near the diagonal $\Delta_{\alpha}$ we divide the sum in (4.50) in two: first, using the estimate (4.46),

$$
\begin{aligned}
\sum_{\substack{i \neq j \\
\left|p_{i}-p_{j}\right|<\alpha}} \iint_{\Delta_{\alpha}} G(x, y) f_{\epsilon}^{i}(x) f_{\epsilon}^{j}(y) & \leq \sum_{\substack{i \neq j \\
\left|p_{i}-p_{j}\right|<\alpha}} C|\ln | p_{i}-p_{j}||\left|f_{\epsilon}^{i}\right|_{L^{1}}\left|f_{\epsilon}^{j}\right|_{L^{1}} \\
& \leq C n_{\epsilon} \ln n_{\epsilon} .
\end{aligned}
$$

Finally, using the estimate (4.46) again,

$$
\begin{aligned}
\sum_{i=1}^{n_{\epsilon}} \iint_{\Delta_{\alpha}} G(x, y) f_{\epsilon}^{i}(x) f_{\epsilon}^{i}(y) & =\sum_{i=1}^{n_{\epsilon}} \int_{B_{\epsilon}(0)} \int_{B_{\epsilon}(0)} G\left(p_{i}+z, p_{i}+w\right) \frac{4}{\epsilon^{4}} d z d w \\
& \leq \sum_{i=1}^{n_{\epsilon}} 4 \int_{B_{1}(0)} \int_{B_{1}(0)}\left(\frac{\left(a\left(r_{*}\right)\right.}{2 \pi} \ln \left[\frac{1}{\epsilon\left|z^{\prime}-w^{\prime}\right|}\right]+C\right) d z^{\prime} d w^{\prime} \\
& =2 \pi a\left(r_{*}\right) n_{\epsilon}|\ln \epsilon|+O\left(n_{\epsilon}\right),
\end{aligned}
$$


recalling that $a_{\gamma}=a$ in the balls $B_{2 \epsilon}\left(p_{i}\right)$. Together, the three previous estimates give

$$
\int_{\mathcal{A}} a|\nabla V|^{2}+\frac{a^{2}}{2 \epsilon^{2}}\left(|V|^{2}-1\right)^{2} \leq \int_{\mathcal{A}} \frac{1}{a_{\gamma}}\left|\nabla h_{\epsilon}\right|^{2}+O\left(n_{\epsilon}\right) \leq 2 \pi a\left(r_{*}\right) n_{\epsilon}|\ln \epsilon|+n_{\epsilon}^{2} A_{*}+o\left(n_{\epsilon}^{2}\right) .
$$

Now let $F_{\theta_{\epsilon}, 0}$ be as in the previous part. We calculate:

$$
\begin{aligned}
\int_{\mathcal{A}} \nabla^{\perp}\left(F_{\theta_{\epsilon}, 0}(|x|)\right) \cdot(i V, \nabla V) & =\int_{\mathcal{A} \backslash \cup B_{2 \epsilon}\left(p_{i}\right)} \nabla\left(F_{\theta_{\epsilon}, 0}(|x|)\right) \cdot \frac{1}{a_{\gamma}} \nabla h_{\epsilon}+o(1) \\
& =\int_{\partial \mathcal{A}} F_{\theta_{\epsilon}, 0} \frac{1}{a_{\gamma}} \frac{\partial h_{\epsilon}}{\partial \nu}-\int_{\cup \partial B_{2 \epsilon}\left(p_{i}\right)} F_{\theta_{\epsilon}, 0} \frac{1}{a_{\gamma}} \frac{\partial h_{\epsilon}}{\partial \nu}+o(1) \\
& =-\sum_{i=1}^{n_{\epsilon}} F_{\theta_{\epsilon}, 0}\left(\left|p_{i}\right|\right) \int_{\partial B_{2 \epsilon}\left(p_{i}\right)} \frac{1}{a} \frac{\partial h_{\epsilon}}{\partial \nu}+o(1) \\
& =-2 \pi \sum_{i=1}^{n_{\epsilon}} F_{0,0}\left(\left|p_{i}\right|\right)+O\left(n_{\epsilon} /|\ln \epsilon|\right) \\
& =-2 \pi n_{\epsilon} a\left(r_{*}\right) K_{0}+O\left(n_{\epsilon} /|\ln \epsilon|\right),
\end{aligned}
$$

where $K_{0}$ is as in Lemma 4.2, and we use (4.17) and (4.26) to replace $F_{\theta_{\epsilon}, 0}$ by $F_{0,0}$. Note also that to pass from the second to the third line we estimate

$$
\begin{aligned}
\mid \int_{\partial B_{2 \epsilon}\left(p_{i}\right)}\left[F\left(|x|-F\left(\left|p_{i}\right|\right)\right] \frac{1}{a} \nabla h \cdot \nu d x \mid\right. & =\mid \int_{B_{2 \epsilon}\left(p_{i}\right)}\left[F(|x|)-F\left(\left|p_{i}\right|\right)\right] \operatorname{div}\left(\frac{1}{a_{\gamma}} \nabla h\right) d x \\
& \quad+\int_{B_{2 \epsilon}\left(p_{i}\right)} \nabla F \cdot \frac{1}{a} \nabla h d x \mid \\
\leq & \frac{2}{\epsilon^{2}} \int_{B_{\epsilon}\left(p_{i}\right)}\left|F(|x|)-F\left(\left|p_{i}\right|\right)\right| d x+ \\
& \left(\int_{B_{2 \epsilon}\left(p_{i}\right)} \frac{1}{a}|\nabla F|^{2}\right)^{1 / 2}\left(\int_{B_{2 \epsilon}\left(p_{i}\right)} \frac{1}{a}\left|\nabla h_{\epsilon}\right|^{2}\right)^{1 / 2} \\
\leq & C \epsilon \sqrt{n_{\epsilon}|\ln \epsilon| .}
\end{aligned}
$$

Together, these estimates give the upper bound,

$$
\begin{aligned}
\mathcal{E}_{\epsilon}(V) & \leq \pi a\left(r_{*}\right) n_{\epsilon}|\ln \epsilon|+\frac{1}{2} n_{\epsilon}^{2} A_{*}-2 \pi n_{\epsilon} a\left(r_{*}\right) K_{0} \Omega+O\left(n_{\epsilon} \ln n_{\epsilon}\right) \\
& =\frac{1}{2} n_{\epsilon}^{2} A_{*}-2 \pi a\left(r_{*}\right) K_{0} \omega_{1} n_{\epsilon} \ln |\ln \epsilon|+O\left(n_{\epsilon} \ln n_{\epsilon}\right) .
\end{aligned}
$$

We recall $n_{\epsilon}=[\ln |\ln \epsilon|]$, and hence

$$
\mathcal{E}_{\epsilon}(V) \leq\left[\bar{A}\left(\omega_{1}\right)+o(1)\right](\ln |\ln \epsilon|)^{2},
$$

with $\bar{A}\left(\omega_{1}\right)=\frac{1}{2} A_{*}-2 \pi a\left(r_{*}\right) K_{0} \omega_{1}$. 
Applying (4.38) and (4.40) to the lower bound (4.31), we have, with $N_{\epsilon}=\Sigma a\left(p_{i}\right) d_{i}$,

$$
\inf \mathcal{E}_{\epsilon} \geq N_{\epsilon}\left(\underline{A}\left(\omega_{1}\right)-o(1)\right) \ln |\ln \epsilon|
$$

with $\underline{A}\left(\omega_{1}\right)=\pi C_{0}-2 \pi K_{0} \omega_{1}$. When $\omega_{1}$ is large enough both $\underline{A}, \bar{A}<0$, and so we have

$$
(-\underline{A}+o(1)) N_{\epsilon} \geq(-\bar{A}-o(1)) \ln |\ln \epsilon|,
$$

and hence we have $N_{\epsilon} \geq C \ln |\ln \epsilon|$. Since $a$ is bounded above we obtain the lower bound for $\Sigma d_{i}$ claimed in (iii) of Theorem 1.2. This completes the proof of Theorem 1.2 $\diamond$

\section{$5 \quad$ When $a(x) \equiv \mathbf{1}_{A}$}

We consider the case of a "square well" potential, that is $a(x)=\mathbf{1}_{A}$ and with a Dirichlet condition imposed on $\partial \mathcal{A}, u \in H_{0}^{1}(\mathcal{A})$. This case has been studied in [Se2] in the case of a disc. In this setting, there is a critical velocity of order $|\ln \epsilon|$ where vortices start to appear. They are first nucleated near the center of the disc. The geometry of the annulus provides, as in the case of the BEC that we have studied a different structure, and we are able to obtain similar results as before: namely, there is a giant vortex in the hole when $\Omega$ is of order one, the degree of the giant vortex depending on $\Omega$, while when $\Omega$ is of order $|\ln \epsilon|$, there is a giant vortex of order $|\ln \epsilon|$ in the hole, and a circle of isolated vortices. The location of the circle is determined by the maximum of $F$. In this case, let $M=\int_{r_{0}}^{R_{0}} s a(s) d s$ and

$$
F(r)=\int_{r}^{R_{0}} a(s)\left(s-\frac{M}{\Lambda_{1} s}\right) d s, \text { with } \Lambda_{1}=\int_{r_{0}}^{R_{0}} \frac{a(s)}{s} d s .
$$

Since $F\left(r_{0}\right)=F\left(R_{0}=0, F\right.$ always attain a maximum in the interval $\left(r_{0}, R_{0}\right)$ and it is at the point $s_{0}^{2}=M / \Lambda_{1}$.

In this case we only need a much narrower barrier around the edges of the annulus. Let $\delta_{\epsilon}=\epsilon^{1 / 3}$, and $\mathcal{A}_{\delta_{\epsilon}}=\left\{x \in \mathcal{A}: \operatorname{dist}(x, \partial \mathcal{A})>\delta_{\epsilon}\right\}, \mathcal{N}_{\epsilon}=A \backslash A_{\epsilon}$ as before. Now

$$
\Omega \int_{\mathcal{N}_{\epsilon}} \eta_{\epsilon}^{2} \mathbf{X} \cdot(i v, \nabla v) \leq \frac{1}{2} \int_{\mathcal{N}_{\epsilon}} \eta_{\epsilon}^{2}|\nabla v|^{2}+O\left(\epsilon^{1 / 3}|\ln \epsilon|^{2}\right)
$$

and we have the improved estimate on the omitted energy,

$$
\tilde{\mathcal{E}}_{\epsilon}(v):=\int_{\mathcal{A}_{\delta_{\epsilon}}}\left\{\frac{1}{2}|\nabla v|^{2}-\Omega \mathbf{X} \cdot(i v, \nabla v)+\frac{1}{4 \epsilon^{2}}\left(|v|^{2}-1\right)^{2}\right\}=E_{\epsilon}(v)+o(1)
$$

The potential function $F_{0,0}(r)$ is defines as before, except now we have

$$
F_{0,0}\left(r_{0}\right)=0=F_{0,0}\left(R_{0}\right), \quad F_{0,0}^{\prime}\left(r_{0}\right)>0, \quad F_{0,0}^{\prime}\left(R_{0}\right)<0 .
$$


The situation near the edges is less delicate, since $a$ and $F_{0,0}$ differ at order one at $r=r_{0}, R_{0}$, and the conclusions of Lemmas 4.2 and 4.3 hold as before.

Returning to the lower estimate (4.31) on the energy, we calculate

$$
\begin{aligned}
o(1) \geq & \tilde{\mathcal{E}}_{\epsilon}(v) \\
& \geq \pi \sum\left|d_{i}\right|\left(|\ln \epsilon|-C_{0} \ln |\ln \epsilon|\right) \\
& \quad-2 \pi \Omega \sum d_{i} F_{\theta_{\epsilon}, \delta_{\epsilon}}\left(\left|p_{i}\right|\right)-2 \pi d_{0} \Omega F_{\theta_{\epsilon}, \delta_{\epsilon}}\left(r_{0}+\delta_{\epsilon}\right)+\frac{1}{2} \int_{\mathcal{A}_{\delta_{\epsilon}} \backslash \cup B_{i}}|\nabla v|^{2},
\end{aligned}
$$

where $d_{0}, \theta_{\epsilon}$ and the vortex balls $\left\{B_{i}\right\}$ are as before, and we again sum over vortex balls in $A_{\epsilon}$. In this case the treatment of the edges is simplified, as

$$
\left|2 \pi d_{0} \Omega F_{\theta_{\epsilon}, \delta_{\epsilon}}\left(r_{0}+\delta_{\epsilon}\right)\right| \leq\left|d_{0}\right| \Omega\left(C \delta_{\epsilon}+\left|\theta_{\epsilon}\right|\right) \leq C\left|d_{0}\right|
$$

In Case I, $\left|d_{0}\right| \leq 2 \sum\left|d_{i}\right|$, we may absorb the term (5.2) into the $\ln |\ln \epsilon|$ term coming from the lower bound of the square gradient inside the balls (modifying the constant $C_{0}$, for example.) In Case II, $\left|d_{0}\right|>2 \sum\left|d_{i}\right|$, we estimate

$$
\frac{1}{2} \int_{\mathcal{A}_{\delta_{\epsilon}} \backslash \cup B_{i}}|\nabla v|^{2} \geq C d_{0}^{2}
$$

as in (4.41) and the proof follows the same lines. In both cases we use the (unweighted) degree counting functions $N_{*}, N_{-}, N_{0}\left(N_{\gamma}\right.$ is unneccessary in this setting) to obtain:

Theorem 5.1 Assume $a(x) \equiv 1$ in $\mathcal{A}, \Omega=\omega_{0}|\ln \epsilon|+\omega_{1} \ln |\ln \epsilon|$, and $u_{\epsilon}$ is a family of energy minimizers as $\epsilon \rightarrow 0$. Then there exist constants $\omega_{0}^{*}, \omega_{1}^{*}$ such that:

(i) if either $\omega_{0}<\omega_{0}^{*}$ or $\omega_{0}=\omega_{0}^{*}$ and $\omega_{1}<\omega_{1}^{*}$, then $u_{\epsilon}$ has no essential vortices in $\mathcal{A}_{\delta_{\epsilon}}$, $\sum\left|d_{i}\right|=0$.

(ii) if $\omega_{0}=\omega_{0}^{*}$ then any vortex in $\mathcal{A}_{\delta_{\epsilon}}$ is localized at the set $\mathcal{C}$ in the following sense: there exists an $M \in \mathbf{N}$ such that

$$
\sum_{d_{i}<0}\left|d_{i}\right|+\sum_{\operatorname{dist}\left(p_{i}, \mathcal{C}\right)>[\ln \epsilon]^{-1 / 2 M}}\left|d_{i}\right|=0
$$

for all $\epsilon$ sufficiently small. Moreover, the sum of the degrees $N_{\epsilon}=\sum\left|d_{i}\right|$ of vortices in $\mathcal{A}_{\delta_{\epsilon}}$ satisfies $N_{\epsilon} \leq C \ln |\ln \epsilon|$ for $C$ independent of $\epsilon$.

(iii) for $\omega_{0}=\omega_{0}^{*}$ there exists $\omega_{1}^{*}>0$ and $C$ (independent of $\epsilon$ ) such that for all $\omega_{1}>\omega_{1}^{*}$, $N_{\epsilon} \geq C \ln |\ln \epsilon|$. 


\section{References}

[ARVK] J. R. Abo-Shaeer, C. Raman, J. M. Vogels , and W. Ketterle, Science 292, 476 (2001).

[AD] A. Aftalion and I. Danaila, Giant vortices in combined harmonic and quartic traps. Phys. Rev. A 69, 033608 (2004).

[ADu] A. Aftalion and Q.Du, Vortices in a rotating Bose-Einstein condensate: Critical angular velocities and energy diagrams in the Thomas-Fermi regime, Phys. Rev. A 64, 063603 (2001).

[AJ] A. Aftalion and R. L. Jerrard, Shape of vortices for a rotating Bose- Einstein condensate, Phys. Rev. A 66, 023611 (2002).

[AR] A. Aftalion and T. Riviere, Vortex energy and vortex bending for a rotating BoseEinstein condensate, Phys. Rev. A 64, 043611 (2001).

[ASSe] A. Aftalion, E. Sandier, S. Serfaty, Pinning phenomena in the Ginzburg-Landau model of superconductivity, J. Math. Pures Appl., vol. 80 (2001), pp. 339-372.

[AnBaPh] N. André, P. Bauman, D. Phillips. Vortex pinning with bounded fields for the Ginzburg-Landau equation. Ann. Inst. H. Poincaré Anal. Non Linéaire 20 (2003), no. 4, 705-729.

[AnSh1] N. André, I. Shafrir, Asymptotic behavior of minimizers for the Ginzburg-Landau functional with weight, Arch. Rat. Mech. Anal., vol. 142 (1998), pp. 45-73.

[AnSh] N. André, I. Shafrir, Minimization of a Ginzburg-Landau type functional with nonvanishing Dirichlet boundary condition, Calc. Var. PDE, 7 (1998), pp. 1-27.

[BBH] F. Bethuel, H. Brezis, F. Hélein. Asymptotics for the minimization of a GinzburgLandau functional. Calc. Var. Partial Differential Equations 1 (1993), no. 2, 123-148.

[BSSD] V. Bretin, S. Stock, Y. Seurin and J. Dalibard, Fast Rotation of a Bose-Einstein Condensate, Phys. Rev. Lett. 92, 050403 (2004).

[BrO] H. Brezis and L. Oswald, Remarks on sublinear elliptic equations, Nonlin. Anal., vol. 10 (1986), pp. 55-64.

[JiMo] S. Jimbo, Y. Morita, Ginzburg-Landau equations and stable solutions in a rotational domain, SIAM J. Math. Anal., vol. 27 (1996), pp. 1360-1385. 
[KTU] K. Kasamatsu, M. Tsubota, M. Ueda, Giant hole and circular superflow in a fast rotating Bose-Einstein condensate, Phys. Rev. B, vol. 66 (2002), 053606.

[L] L. Lassoued, Asymptotics for a Ginzburg-Landau model with pinning, Comm. Appl. Nonlinear Anal., vol. 4 (1997), pp. 27-58.

[LM] L. Lassoued and P. Mironescu, Ginzburg-Landau type energy with discontinuous constraint, J. Anal. Math., vol. 77 (1999), pp. 1-26.

[MCBD] K. W. Madison, F. Chevy, V. Bretin, and J. Dalibard, Stationary States of a Rotating Bose-Einstein Condensate: Routes to Vortex Nucleation Phys. Rev. Lett. 86, 4443-4446 (2001).

[RBD] P. Rosenbusch, V. Bretin and J. Dalibard, Dynamics of a Single Vortex Line in a Bose-Einstein Condensate, Phys. Rev. Lett. 89, 200403 (2002)

[RuSt] J. Rubinstein, P. Sternberg, Homotopy classification of minimizers of the GinzburgLandau energy and the existence of permanent currents, Comm. Math. Phys., vol. 179 (1996), pp. 257-263.

[Sa] E. Sandier, Lower Bounds for the Energy of Unit Vector Fields and Applications, J. Functional Analysis, vol. 152 (1998), 379- 403.

[SSe1] E. Sandier and S. Serfaty, Global Minimizers for the Ginzburg-Landau Functional Below the First Critical Magnetic Field, Annales IHP, Analyse Non Linéaire, vol. 17 (2000), 119-145.

[SSe2] E. Sandier and S. Serfaty, On the energy of type-II superconductors in the mixed phase. Rev. Math. Phys. 12 (2000), no. 9, 1219-1257.

[SSe3] E. Sandier and S. Serfaty, A Rigorous Derivation of a Free-Boundary Problem Arising in Superconductivity, Ann. Scient. Éc. Norm. Sup., $4^{e}$ série, t. 33 (2000), 561-592.

[SSe4] E. Sandier and S. Serfaty Ginzburg-Landau Minimizers Near the First Critical Field Have Bounded Vorticity, preprint (2001).

[SSo] E. Sandier and M. Soret, $\mathbf{S}^{1}$-Valued Harmonic Maps with High Topological Degree: Asymptotic Behavior of the Singular Set, Potential Analysis, vol. 13 (2000), pp. 169184. 
[Se2] S. Serfaty, On a Model of Rotating Superfluids, ESAIM: Control, Optim., Calc. Var., vol. 6 (2001), pp. 201-238.

[SBCD] S. Stock, V. Bretin, F. Chevy and J. Dalibard, Shape oscillation of a rotating BoseEinstein condensate cond-mat/0311099 (2003). 